\title{
Power and energy scaling of rod-type cryogenic Yb:YLF regenerative amplifiers
}

\author{
Umit Demirbas, ${ }^{1,2, *}$ (1) Huseyin Cankaya, ${ }^{1,3,4}$ (iD Mikhail Pergament, ${ }^{1}$ and \\ FrANZ X. KÄRTNER ${ }^{1,3,4}$ (i) \\ ${ }^{1}$ Center for Free-Electron Laser Science, Deutsches Elektronen-Synchrotron DESY, Notkestraße 85, 22607 Hamburg, Germany \\ ${ }^{2}$ Laser Technology Laboratory, Department of Electrical and Electronics Engineering, Antalya Bilim University, Antalya, Turkey \\ ${ }^{3}$ Physics Department, University of Hamburg, Luruper Chaussee 149, 22761 Hamburg, Germany \\ ${ }^{4}$ The Hamburg Centre for Ultrafast Imaging, Luruper Chaussee 149, 22761 Hamburg, Germany \\ *Corresponding author: uemit.demirbas@cfel.de
}

Received 30 January 2020; revised 30 April 2020; accepted 3 May 2020; posted 4 May 2020 (Doc. ID 389548); published 27 May 2020

\begin{abstract}
We numerically investigate the power and energy scaling potential of cryogenic Yb:YLF regenerative amplifiers in rod geometry. Our approach is based on solving the coupled set of equations describing thermal behavior of the material and its effect on spectroscopic properties, gain, and overall amplification. The approach is first benchmarked with earlier experimental data. By carefully analyzing the sensitivity of the system to operation parameters, we see that the relatively low gain nature of the Yb:YLF and the onset of thermal effects are the main factors that limited the performance in earlier experimental work. We show that usage of dual-rod geometry promises much improved performance. Specifically, we demonstrate that sub-250 fs pulses with an average power of up to $270 \mathrm{~W}$ and a peak power above $500 \mathrm{GW}$ can be extracted directly from a single-stage Yb:YLF regenerative amplifier employing dual Yb:YLF rods. We further show that by adjusting the spot size in the regenerative amplifier, one can operate the amplifier in either high-energy mode $(>100 \mathrm{~mJ}$ at $1 \mathrm{kHz})$ or high-average-power mode $(>25 \mathrm{~mJ}$ at $10 \mathrm{kHz}$, with $>250 \mathrm{~W}$ ). We also discuss pros and cons of $\mathrm{Yb}$ :YLF with respect to $\mathrm{Yb}$ :YAG, and underline the need for measurement of population and photo-elastic-effect-induced lensing in Yb:YLF to obtain a better understanding of Yb:YLF systems. The findings presented in this work can be used for the design and development of next-generation high-average and peak-power Yb:YLF amplifier systems. (C) 2020 Optical Society of America
\end{abstract}

\section{INTRODUCTION}

$\mathrm{Yb}: Y A G$ gain media combine the simple and favorable energy level structure of the $\mathrm{Yb}$ ion with the thermo-mechanical strength of the YAG host, and as a result, it enables development of leading-edge high-power and high-energy laser/amplifier systems [1-9]. On the other hand, the narrow and steep gain profile of $Y b$ :YAG results in strong gain narrowing that is hard to compensate for (Fig. 1). As a consequence, the pulse widths obtainable from Yb:YAG amplifiers are limited to $500 \mathrm{fs}$ to $1 \mathrm{ps}$ at room temperature and to a few picoseconds at cryogenic temperatures. Moreover, the thermo-optic coefficient $(\mathrm{dn} / \mathrm{dT})$ and the polarizability difference parameter $(\Delta \alpha$ : scale of population lensing) are both positive in Yb:YAG, resulting in an earlier onset of thermal lensing, especially in rod geometry.

As an interesting alternative, the Yb:YLF gain medium shows broad emission bands even at cryogenic temperatures [10-14]. Specifically, the $E / / a$ axis emission of the uniaxial Yb:YLF gain medium, centered at around $1016 \mathrm{~nm}$, has a full-width half-maximum (FWHM) of about $10 \mathrm{~nm}$ (Fig. 1). The emission spectrum is also rather smooth/flat, which minimizes gain narrowing upon amplification [15]. In carefully designed systems, amplified pulse widths below 250 fs should become feasible [16]. Furthermore, earlier experimental work indicates that $\mathrm{Yb}$ :YLF combines a negative thermo-optic coefficient with positive population and/or photo-elastic-effect-induced lensing, and when combined, this results in a relatively small positive thermal lens [15]. As a result, compared to Yb:YAG, this provides a lower amount of beam distortion per heat load. Furthermore, efficient lasing can be obtained in heavily doped Yb:YLF samples as well $[17,18]$. On the other hand, as a disadvantage, in general the thermo-mechanical strength of $\mathrm{Yb}: \mathrm{YLF}$ is not as high as for Yb:YAG (see Table 1 in [1] for a detailed comparison). Moreover, the broadband $E / / a$ emission around $1016 \mathrm{~nm}$ has a relatively small emission cross section value (14 times lower when compared to cryogenic $\mathrm{Yb}: \mathrm{YAG}$ ), which results in a relatively low gain and high saturation fluence $\left(\sim 14 \mathrm{~J} / \mathrm{cm}^{2}\right)$ system that is sensitive to cavity losses. Hence, optimization of Yb:YLF amplifiers is difficult, especially for the broadband $E / / a$ axis transition. 


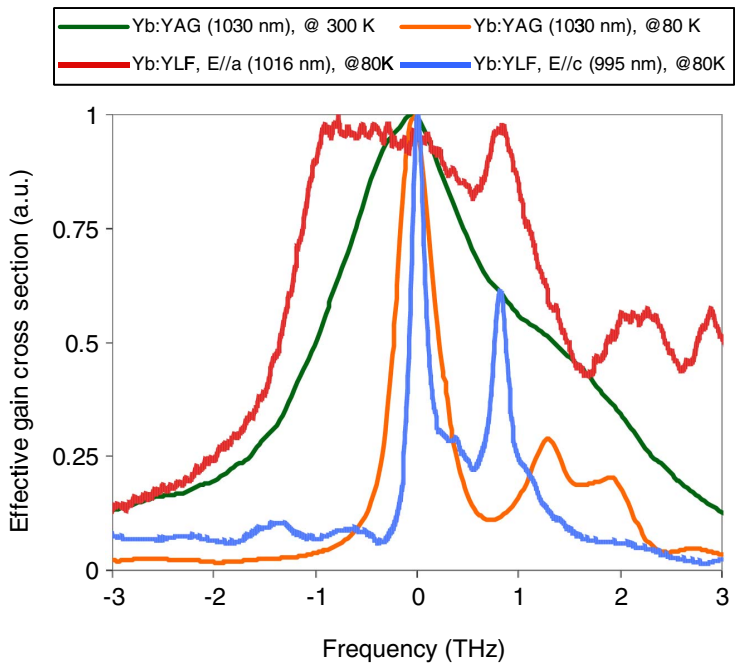

Fig. 1. Comparison of effective gain profile of cryogenic Yb:YLF with Yb:YAG in frequency domain. The centers of the gain spectra are shifted to base band for better comparison. The graph contains the broadband $1016 \mathrm{~nm}$ emission band of $\mathrm{Yb}: \mathrm{YLF}(E / / a, 80 \mathrm{~K})$, the narrowband $995 \mathrm{~nm}$ emission band of $\mathrm{Yb}: \mathrm{YLF}(E / / c, 80 \mathrm{~K})$, and $1030 \mathrm{~nm}$ band of $\mathrm{Yb}: \mathrm{YAG}$ at room and cryogenic temperatures. An inversion level of $25 \%$ is assumed for room-temperature Yb:YAG, and all curves are shown in normalized units.

The amplification potential of Yb:YLF has already been explored, but only in a few studies [19-23]. Using regenerative amplifiers, pulses with $30 \mathrm{~mJ}$ energy at $20 \mathrm{~Hz}$ [19], $20 \mathrm{~mJ}$ energy with up to $3.5 \mathrm{kHz}[15]$, and up to $5 \mathrm{~mJ}$ energy at $10 \mathrm{kHz}$ $[15,20]$ have been demonstrated using the broadband $E / / a$ axis of $\mathrm{Yb}: Y L F$. In multipass amplification geometry, pulse energies up to $190 \mathrm{~mJ}$ have been reported at $10 \mathrm{~Hz}$ [23], and a pulse energy of $10 \mathrm{~mJ}$ was demonstrated at $10 \mathrm{kHz}$ [21]. The strong and narrow gain peak around $995 \mathrm{~nm}$ in the $E / / c$ axis of the medium has also been recently explored to demonstrate up to $40 \mathrm{~W}$ of average power in a multipass amplifier seeded by a cw source (Fig. 1 shows the emission profile for this transition as well) [22]. However, the literature lacks a detailed study investigating the limits of $\mathrm{Yb}: \mathrm{YLF}$ regenerative amplifiers in rod geometry.

In this work, we numerically explore the power and energy scaling potential of Yb:YLF regenerative amplifiers in rod geometry in detail. We show that significant improvements beyond the earlier $\mathrm{Yb}$ :YLF regenerative amplification results in the literature are possible: (i) by utilizing an optimized broadband $(>15 \mathrm{~nm})$ seed source with adequate stretching and (ii) by exploiting a dual-rod geometry. The adequately stretched $(\sim 2 \mathrm{~ns})$ broadband seed permits extraction of higher energies by increasing the laser-induced-damage threshold (LIDT). The dual-rod geometry significantly boosts the gain, and enables operation of the regenerative amplifier at higher repetition rates and average power. Specifically, sub-250 fs pulses with an average power up to $270 \mathrm{~W}$ and a peak power up to $600 \mathrm{GW}$ look feasible by using the $E / / a$ axis of Yb:YLF. By adjusting the spot size employed, the system could be optimized either as a high-energy and -peak power tool at moderate repetition rates $(\sim 100 \mathrm{~mJ}$ at $1 \mathrm{kHz})$, or as a high-average-power source
(>25 mJ at $10 \mathrm{kHz}$, with $>250 \mathrm{~W}$ ). We hope the findings presented in this work will be useful to laser engineers and scientists working with $\mathrm{Yb}: \mathrm{YLF}$ systems.

The paper is organized as follows. Section 2 presents details on the investigated regenerative amplifier geometries, and briefly presents the numerical model that has been applied. In Section 3, we discuss the estimated thermo-mechanical behavior of the Yb:YLF rod under thermal load. Section 4 summarizes calculated amplifier performance in detail. Finally, in Section 5, we conclude with a brief discussion.

\section{DETAILS OF THE INVESTIGATED YB:YLF REGENERATIVE AMPLIFIER GEOMETRY AND NUMERICAL MODEL}

\section{A. Investigated Regenerative Amplifier Geometries}

In our analysis, we have investigated two different but quite similar cryogenic Yb:YLF regenerative amplifier cavities. The first one, shown in Fig. 2(a), is almost identical to what we have explored in a recent experimental campaign [15]. A state-of-theart laser diode module provides up to $2 \mathrm{~kW}$ of pump power at a central wavelength of $960 \mathrm{~nm}$, from a $600 \mu \mathrm{m}$ core diameter fiber, with an estimated $\mathrm{M}^{2}$ of 220 . The pump light is collimated and imaged inside the gain medium using a set of appropriate lenses ( $\mathrm{f} 1-\mathrm{f} 3$ ). The $1 \% \mathrm{Yb}$-doped $\mathrm{Yb}$ :YLF gain medium is $2 \mathrm{~cm}$ long and has $3 \mathrm{~mm}$ long un-doped end cap diffusion bonded sections on both ends (total crystal length: $L=26 \mathrm{~mm}$ ), and occupies a cross section of $10 \mathrm{~mm}(\mathrm{H}$ : height $) \times 15 \mathrm{~mm}$ (W: width). The crystal is indium bonded from the top side to a multi-stage pyramidal cold head, which was cooled to cryogenic
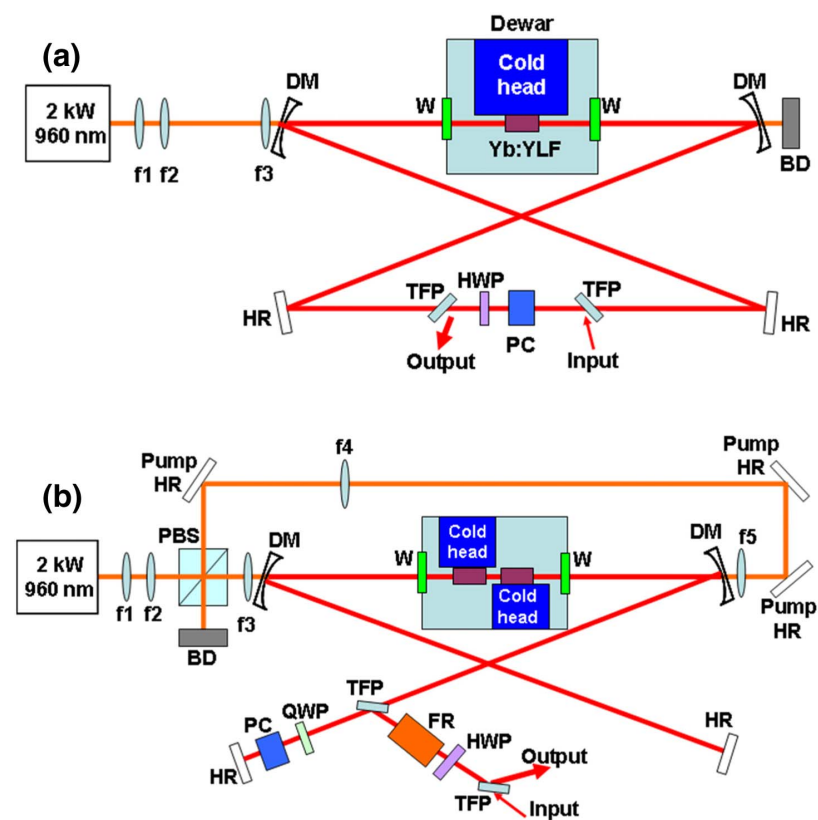

Fig. 2. Schematics of the cryogenic Yb:YLF regenerative amplifiers that have been modeled in the simulations. (a) Ring cavity with a single $\mathrm{Yb}$ :YLF crystal and (b) standing wave cavity employing two Yb:YLF crystals. DM, dichroic mirror; f1-f5, lens; W, antireflection coated window; PC, Pockels cell; QWP, quarter-wave plate; HWP, half-wave plate; TFP, thin-film polarizer; PBS, polarizing beam splitter cube; FR, Faraday rotator; $\mathrm{HR}$, high-reflector mirror; $\mathrm{BD}$, beam dump. 

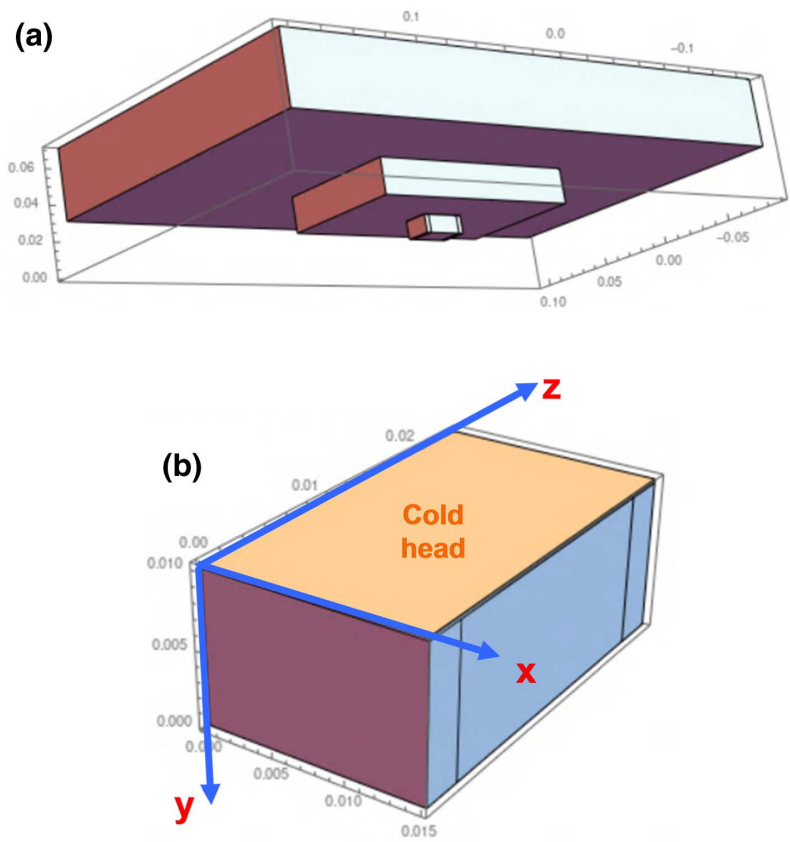

Fig. 3. (a) Yb:YLF crystal bonded to the heat sink via indium bonding. The heat sink is designed in pyramid structure to improve heat extraction efficiency via heat spreading. The top part of the heat sink is in direct contact with liquid nitrogen. (b) Simplified geometry used in thermal simulations, which contains only the Yb:YLF crystal and the indium bonding layer.

temperatures by liquid nitrogen (Fig. 3). One side cooling creates asymmetry in cooling; on the other hand, it is still preferred to ease escape of unused inversion via spontaneous emission. The pyramidal structure enables efficient heat spreading and boosts the heat extraction capability of the structure [24,25]. The bow-tie type regenerative ring cavity consists of two curved dichroic mirrors (DMs), and two flat high reflectors (HRs). Two thin-film polarizer's (TFPs), a half-wave plate (HWP), and a Pockels cell (PC) were used for seeding of the amplifier. The system is seeded by stretched pulses from a fiber front-end system similar to what is described in Ref. [26].

The second regen cavity that is explored is quite similar but employs two Yb:YLF crystals with identical specs to boost up the gain of the system. In this case, the pump is split into two equal parts using a polarizing beam splitter (PBS) cube, and the Yb:YLF crystals are pumped with equal amounts of pump power from each side. The estimated pump absorption of a single crystal is $>80 \%-90 \%$ even under saturation conditions; hence, there is little crosstalk between the Yb:YLF crystals via the remaining unabsorbed pump light. The second regenerative amplifier cavity shown is an X-type standing wave cavity, where the PC is used in quarter-wave mode to couple in/out the seed beam. Hence, we assume that the simulated cavities are either ring or standing wave cavities, employing either a single- or double-gain element, and the cavity type should be chosen based on the spot size required. Basically, PCs with larger aperture are available in quarter-wave mode; hence, standing wave cavities might be the only solution for large-spot-sized systems. In terms of our numerical analysis, ring and standing wave cavities are equivalent.

\section{B. Thermo-Mechanical Model}

In our analysis, we first investigated the thermo-mechanical behavior of the $\mathrm{Yb}$ :YLF crystals under thermal load, since the temperature of the gain element could significantly influence effective gain. In our earlier work, we saw experimentally that the maximum average pump power that could be handled effectively by a single Yb:YLF rod laser/amplifier $\left(P_{\text {abs, max }}\right)$ was roughly around $[1,15]$

$$
P_{\mathrm{abs}, \max }=350 \mathrm{~W}+\frac{1}{2} P_{\mathrm{out}},
$$

where $P_{\text {out }}$ is the average output power extracted out of the system. As an example, for a single-crystal regenerative amplifier system with $100 \mathrm{~W}$ average power, the estimated maximum applicable absorbed pump power is around $400 \mathrm{~W}$. For the dual-rod system, we assume that the total absorbed pump power could be roughly doubled (this issue will be elaborated in more detail later). Note that we believe this restraint is a result of the boiling liquid nitrogen boundary condition, where the Leidenfrost effect limits the heat extraction capability of the system [27-29]. This limitation could potentially be improved by surface modification of the inner surface of the heat sink that is in direct contact with liquid nitrogen [30,31].

For an initial 3D simulation of temperature and resulting thermal lensing inside the Yb:YLF rod, a simplified model of the crystal geometry consisting of only the Yb:YLF crystal and the indium bonding layer was used for the sake of simplicity [Fig. 3(b)]. We further assumed cw pumping conditions for the regenerative amplifier, which is the standard case for highrepetition-rate operation. Then, in steady state, the temperature distribution inside the crystal, $T(x, y, z)$, is given by Ref. [32]:

$$
\nabla \cdot[k(x, y, z, T) \nabla T(x, y, z)]+Q(x, y, z)=0,
$$

where $k(x, y, z, T)$ is the temperature-dependent thermal conductivity in different regions, and $Q(x, y, z)$ is the heat source density (thermal load per unit volume in the units of $\mathrm{W} / \mathrm{m}^{3}$ ). The temperature dependence of thermal conductivity of YLF [33], 1\% doped Yb:YLF [33], and indium [34] layers are modeled via

$$
\begin{gathered}
k_{\mathrm{YLF}, a}(T) \cong 21800 T^{-1.48}, \\
k_{\mathrm{YLF}, c}(T) \cong 33000 T^{-1.49}, \\
k_{1 \% \mathrm{Yb}: \mathrm{YLF}, a}(T) \cong 14300 T^{-1.41}, \\
k_{1 \% \mathrm{Yb}: \mathrm{YLF}, c}(T) \cong 21200 T^{-1.42}, \\
k_{\text {ind }}(T) \cong 120.6-0.01465 T+0.0001 T^{2} .
\end{gathered}
$$

In Eqs. (3)-(7), $k$ is the thermal conductivity in units of $\mathrm{W} / \mathrm{mK}$, and $T$ is temperature in units of $\mathrm{K}$. Also the $1 \% \mathrm{Yb}: \mathrm{YLF}$ thermal conductivity data in Eqs. (5) and (6) are estimated by linearly interpolating the data of un-doped YLF and 5\% doped Yb:YLF in Ref. [33]. At a temperature of $120 \mathrm{~K}$, the thermal conductivity of $1 \% \mathrm{Yb}$-doped $\mathrm{Yb}: \mathrm{YLF}$ is estimated as $16.6 \mathrm{~W} / \mathrm{mK}$ and 
23.6 W/mK in $a$ and $c$ axes, respectively. Note that here we specified the values at $120 \mathrm{~K}$, since as we will discuss in the next section, this is our estimated average temperature within the pumped volume of the Yb:YLF crystal.

The heat source density is calculated via [35]

$$
\begin{aligned}
Q(x, y, z)= & \frac{2(\mathrm{FTL}) P_{\text {in }} \alpha_{\mathrm{eff}}}{\pi w_{p 0}^{2}(z)} \operatorname{Exp}\left[-\frac{2\left(x^{2}+y^{2}\right)}{w_{p 0}^{2}(z)}\right] \\
& \times \operatorname{Exp}\left[-\alpha_{\mathrm{eff}} z\right]
\end{aligned}
$$

where FTL is the fractional thermal load, and $P_{\text {in }}$ is the incident pump power. In Eq. (8), $w_{p 0}(z)$ is the pump mode radius at position $z$ within the crystal and is calculated using

$$
\omega_{p 0}(z)=\omega_{0} \sqrt{1+\left(\frac{z-L / 2}{z_{r}}\right)^{2}},
$$

where $z_{R}$ is the Rayleigh range for the pump beam, which is estimated with

$$
z_{r}=\frac{1}{M^{2}} \frac{\pi \omega_{0}^{2}}{\lambda_{p}}
$$

In Eq. (10), $\lambda_{p}$ is the pump wavelength (960 nm), and $\mathrm{M}^{2}$ is the beam quality factor of the pump beam $(\sim 220)$. In the thermal simulations, the total absorbed pump power is taken as $400 \mathrm{~W}$, along with a pump beam waist $\left(w_{0}\right)$ of $1 \mathrm{~mm}$. In Eq. (8), $\alpha_{\text {eff }}$ is the effective absorption coefficient of the gain medium that is estimated from the experimentally measured values considering: (i) spectral match between the pump spectra and $\mathrm{Yb}: \mathrm{YLF}$ absorption profile, and (ii) pump saturation effects [36].

Figure 4 shows estimated distribution of the absorbed pump power within the Yb:YLF crystal volume. In our analysis, we have assumed that the FTL is 1.5 times higher than the quantum defect (QD) due to undesired factors such as radiative trapping and existence of nonradiative sites [37]:

$$
\mathrm{FTL}=1.5(\mathrm{QD})=1.5\left(1-\frac{\lambda_{p}}{\lambda_{l}}\right),
$$

where $\lambda_{l}$ is the central laser/amplifier wavelength, which is expected to be around $1016 \mathrm{~nm}$ for amplification in the $E / / a$ axis, corresponding to a QD of 5.5\%, and an assumed FTL of around $8.3 \%$.

A boundary condition in the form of a Newton-type of cooling is assumed in thermal calculations, where we have simply assumed that the $\mathrm{Yb}: \mathrm{YLF}$ is cooled from the top surface of the indium bonding located at $y=-h_{\text {ind }}[32,35]$ :

$$
\left.\frac{\partial T(x, y, z)}{\partial y}\right|_{y=-h_{\text {ind }}}=\frac{H_{\text {htc }}}{k_{\text {ind }}}\left(T_{\mathrm{LN}}-T\right) .
$$

The Yb:YLF rod and indium bonding layer contact is assumed to be located at $y=0$ [Fig. 3(b)], and the thickness of the indium $\left(h_{\text {ind }}\right)$ is taken as $250 \mu \mathrm{m}$. Thermal insulation is assumed for all the other surfaces. In Eq. (12), $T_{\mathrm{LN}}=77 \mathrm{~K}$ is the temperature of liquid nitrogen, and $H_{\mathrm{htc}}$ is the heat transfer coefficient, which is taken as $20 \mathrm{~kW} / \mathrm{m}^{2}$. Note that the heat transfer coefficient value we have assumed here is higher than what can be
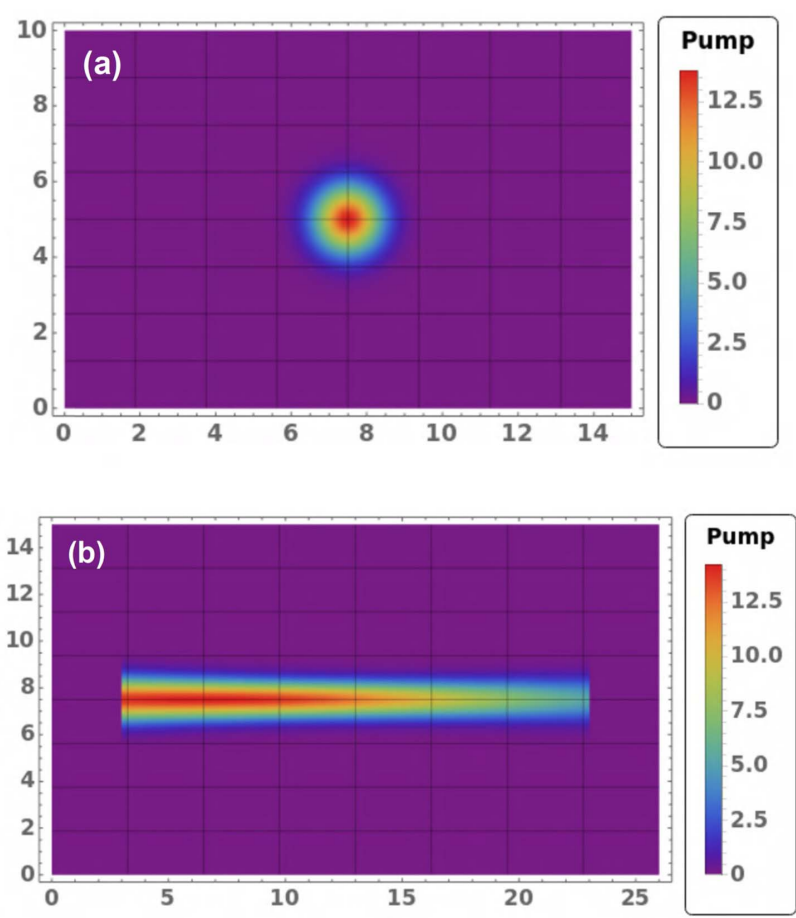

Fig. 4. Pump beam profile incident on the Yb:YLF crystal [length (L): $20+3+3 \mathrm{~mm}$, height $(\mathrm{H}): 10 \mathrm{~mm}$, width (W): $15 \mathrm{~mm}$ ]. The pump beam waist is taken as $1 \mathrm{~mm}$. The dimensions are in $\mathrm{mm}$, and the pump density is in units of $\mathrm{kW} / \mathrm{cm}^{3}$. The calculation was performed for a total absorbed pump power of $400 \mathrm{~W}$.

usually achieved in a regular direct liquid nitrogen boundary $\left(10 \mathrm{~kW} / \mathrm{m}^{2}\right.$ [27-29,35]), and the assumed improvement is due to the advantages of pyramidal design of the cold head [24,25]. Recent experimental results confirm that nanoscale surface modification could further be used to improve the heat transfer efficiency [30,31].

Once the temperature distribution $[T(x, y, z)]$ is calculated, the volume element displacement vector function $\vec{U}$ is calculated using [32]

$$
\nabla^{2} \vec{U}+\frac{1}{1-2 v} \nabla(\nabla \cdot \vec{U})=\frac{2(1+v)}{1-2 v} \alpha \nabla T,
$$

where $v$ is the Poisson's ratio (taken as 0.33 for Yb:YLF [38] and 0.435 for indium [39]), and $\alpha$ is the thermal expansion coefficient and is calculated via $[33,40]$

$$
\begin{gathered}
\alpha_{\mathrm{YLF}, a}(T) \cong-8+0.118 T-0.000146 T^{2}, \\
\alpha_{\mathrm{YLF}, c}(T) \cong-4.75+0.094 T-0.00015 T^{2}, \\
\alpha_{\text {ind }}(T) \cong 16.24+0.085 T-0.000108 T^{2} .
\end{gathered}
$$

In Eqs. (14)-(16), $\alpha$ is in units of $\mathrm{ppm} / \mathrm{K}\left(10^{-6} \mathrm{~m} / \mathrm{K}\right)$, and $\mathrm{T}$ is again in units of Kelvin. At a temperature of $120 \mathrm{~K}$, the thermal expansion coefficient of $\mathrm{Yb}$ :YLF has estimated values of $4.1 \mathrm{ppm} / \mathrm{K}$ and $4.4 \mathrm{ppm} / \mathrm{K}$ in $a$ and $c$ axes, respectively. While calculating the displacement vector, we assume that the back side of the indium solder that is in contact with the bulky cold 
head metal is positioned at $y=-h_{\text {ind }}$ and is stationary. We also carefully define specific parameters of Yb:YLF, YLF, and indium solder in appropriate geometric regions, and the anisotropy of $\mathrm{Yb}: \mathrm{YLF}$ is also taken into account. At this point, the optical path difference (OPD) variations for the beam passing through the $\mathrm{Yb}: \mathrm{YLF}$ rod are estimated using $[32,41]$

$$
\begin{aligned}
\operatorname{OPD}(x, y)= & \left(n_{o}-1\right) \Delta L(x, y) \\
& +\frac{\partial n}{\partial T} \int_{0}^{L}\left[T(x, y, z)-T_{\mathrm{LN}}\right] \mathrm{d} z,
\end{aligned}
$$

where the first term corresponds to changes in optical path length due to deformations in the rod's surface, and the second term corresponds to the variation in the index of refraction with temperature. The temperature dependence of the thermal coefficient of refractive index for YLF is calculated using [33]

$$
\begin{aligned}
& \frac{d n}{d T_{\mathrm{YLF}, a}}(T) \cong-0.104+0.00105 T-0.000053 T^{2}, \\
& \frac{d n}{d T_{\mathrm{YLF}, c}}(T) \cong-0.039-0.00155 T-0.0000217 T^{2} .
\end{aligned}
$$

Note that in Eqs. (18) and (19), $\mathrm{T}$ is in $\mathrm{K}$, and at $120 \mathrm{~K}, \mathrm{dn} / \mathrm{dT}$ has values of around $-0.75 \times 10^{-6} / \mathrm{K}$ and $-2.2 \times 10^{-6} / \mathrm{K}$ in $a$ and $c$ axes, respectively. We could not account for OPD due to population/electronic lensing in this study, because to our knowledge, the polarizability difference parameter $(\Delta \alpha)$ is not yet measured for $\mathrm{Yb}: \mathrm{YLF}$ [42]. We are also ignoring the OPD created by strain-induced birefringence, since to our knowledge, photo-elastic parameters for YLF are not known [32,43,44].

\section{Amplification Model}

In the modeling of the regenerative amplifier performance, we used the well-known Frantz-Nodvik equations [45-47]. In this model, if the duration of the amplified pulse and the time of flight through the amplifying medium are short compared to the fluorescence lifetime of the gain medium, then one can neglect spontaneous emission and use the following analytical equation for the modeling of the amplification process in the amplifier:

$$
J_{\text {out }}=J_{\text {sat }} \operatorname{Ln}\left[1+e^{g 0}\left(e^{\frac{J_{\text {in }}}{\int_{\text {sat }}}}-1\right)\right],
$$

where $J_{\text {in }}\left(J_{\text {out }}\right)$ is the incident (output) pulse fluence, and $g_{0}$ is the small signal fractional gain. The incident (output) pulse fluence could be determined simply by $J_{\text {out }}=E_{\text {out }} / A_{\text {eff }}$ and $J_{\text {in }}=E_{\text {in }} / A_{\text {eff }}$, where $A_{\text {eff }}$ is the effective pump/laser beam area $\left(\pi w_{l}^{2}\right)$, and $E_{\text {in }}$ and $E_{\text {out }}$ are the input and output pulse energies, respectively. The remaining parameters in Eq. (20) can be calculated using the following identities:

$$
\begin{gathered}
J_{\text {sat }}=\frac{h c}{\lambda_{l}\left(\sigma_{\mathrm{em}}+\sigma_{\mathrm{ab}}\right)}=\frac{E_{\lambda_{l}}}{\left(\sigma_{\mathrm{em}}+\sigma_{\mathrm{ab}}\right)}, \\
J_{\text {sto }}=\frac{E_{\mathrm{abs}}}{\mathrm{A}_{\mathrm{eff}}} \frac{\lambda_{p}}{\lambda_{l}}, \\
g_{0}=\frac{J_{\text {sto }}}{J_{\mathrm{abs}}} \cong \frac{E_{\mathrm{abs}}}{E_{\lambda_{l}}} \frac{\lambda_{p}}{\lambda_{l}} \operatorname{MMF} \frac{\left(\sigma_{\mathrm{em}}+\sigma_{\mathrm{ab}}\right)}{A_{\text {eff }}},
\end{gathered}
$$

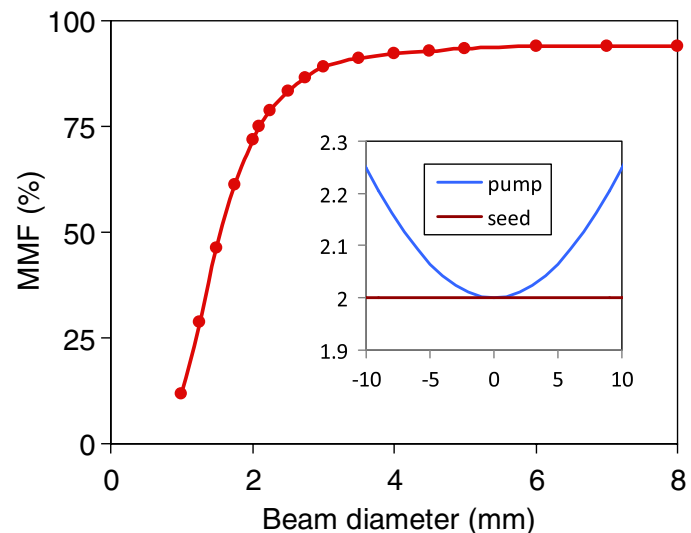

Fig. 5. Estimated mode-matching factor (MMF) between the pump and resonator beams as a function of pump/seed spot size employed in the regen. The estimation was performed for a $2 \mathrm{~cm}$ long $Y b: Y L F$ gain element assuming homogeneous distribution of inversion along the crystal length. The beam qualities ( $\mathrm{M}^{2}$ factors) of the pump and laser beams are taken as 250 and one, respectively. As an example, inset shows the mode-mismatch between the pump and seed beams as they propagate through the Yb:YLF crystal for a sample beam waist of $1 \mathrm{~mm}$ (corresponds to a minimum beam diameter of $2 \mathrm{~mm}$ at the center of the crystal).

where $J_{\text {sto }}$ is the stored energy density, $J_{\text {sat }}$ is the saturation fluence of the gain medium, $b$ is Planck's constant, $c$ is the speed of light, $E_{\text {abs }}$ is the absorbed pump energy, $E_{\lambda_{l}}$ is the photon energy at the laser wavelength, $\sigma_{\mathrm{ab}}\left(\sigma_{\mathrm{em}}\right)$ is the effective absorption (emission) cross section at the amplifier wavelength, and MMF is the mode-matching factor between the pump and circulating seed modes.

In our simulations, the MMF is estimated as a function of pump/laser spot size employed, by considering the propagation of pump and laser/amplifier beams within the $2 \mathrm{~cm}$ long Yb:YLF crystal (Fig. 5). In the analysis, the beam waists of pump and laser/seed beams are assumed to be the same (mode matched) at the center of the gain element. MMF considers the mode mismatch between the two beams as they propagate along the crystal. Due to a significant difference in their beam quality factor, the pump beam has a significantly shorter Rayleigh range compared to the seed/laser beam. As can be seen, the estimated MMF for a $1 \mathrm{~mm}$ beam waist is around $70 \%$, and reduces sharply for smaller beam sizes.

An accurate solution to the amplification process requires knowledge of the temperature dependence of effective emission and absorption cross sections of Yb:YLF around $1016 \mathrm{~nm}$ for the $E / / a$ axis. We have used the known energy-level scheme of $\mathrm{Yb}: \mathrm{YLF}$ along with the measured spectroscopic data at $77 \mathrm{~K}$ and $300 \mathrm{~K}$ to obtain the following Boltzmann-distribution-based occupation formulas for a rough estimation of temperature variation of effective emission/absorption cross sections in the $77-150 \mathrm{~K}$ range (for $E / / a$ axis at $1016 \mathrm{~nm}$ ) $[12,48,49]$ :

$$
\begin{aligned}
\sigma_{\mathrm{em}}(T) \cong & 0.75 \times 10^{-20}\left[1-3.3 \times 10^{-3}(T-77)\right. \\
& \left.+7.2 \times 10^{-6}(T-77)^{2}\right], \\
\sigma_{\mathrm{ab}}(T) \cong & 0.04 \times 10^{-20}\left[0.03+0.000025(T-77)^{2}\right] .
\end{aligned}
$$


At a temperature of $120 \mathrm{~K}$, the emission and absorption cross sections of $\mathrm{Yb}: \mathrm{YLF}$ at $1016 \mathrm{~nm}$ have an estimated value of around $0.65 \times 10^{-20} \mathrm{~cm}^{2}$ and $0.002 \times 10^{-20} \mathrm{~cm}^{2}$, respectively. We see that for lasing at this transition, $\mathrm{Yb}: \mathrm{YLF}$ is almost a four-level system at cryogenic temperatures. As a reminder, since $\sigma_{\mathrm{em}} \gg\left(\sigma_{\mathrm{ab}} \sim 0\right)$, average fractional population inversion $(\beta)$ of the material could be roughly estimated using Eq. (23) via

$$
\beta \cong \frac{g_{0}}{L_{g}\left(N_{\mathrm{Yb}}\right) \sigma_{\mathrm{em}}},
$$

where $N_{\mathrm{Yb}}$ is the number density of the active $\mathrm{Yb}$ ions in the Yb:YLF sample, and $L_{g}$ is the actively doped length of the gain material. For an isolated square pump pulse with a peak power of $P_{\text {peak }}$ and a pulse width of $T$, the useful absorbed energy by the gain medium from this single pulse $\left(E_{\mathrm{abs}, s}\right)$ is

$$
E_{\mathrm{abs}, s}=P_{\mathrm{abs}} T \frac{\left[1-e^{-\frac{T}{\tau}}\right] \tau}{T}=P_{\mathrm{abs}} \tau\left[1-e^{-\frac{T}{\tau}}\right],
$$

where $P_{\text {abs }}$ and $\tau$ are the absorbed pump power and fluorescence lifetime of the laser material, respectively. The absorbed pump power $\left(P_{\mathrm{abs}}\right)$ can be estimated by multiplying the pump peak power $\left(P_{\text {peak }}\right)$ by the overall effective absorption (Abs) of the gain material. As mentioned earlier, effective absorption of the material depends on the spectral width and central wavelength of the pump diode and how it matches to the absorption profile of the gain medium. Moreover, the pump saturation effect might also be present, and hence the pump beam diameter (the pump fluence) and the intracavity circulating regen power will also influence the effective absorption amount. A simple Beer-Lambert-type formula could be used for simplification of modeling, by defining an effective absorption coefficient $\left(\alpha_{\text {eff }}\right)$ parameter that matches the experimentally measured absorption data for the different configurations [1,15].

Equation (27) above shows that for pump pulses significantly shorter than lifetime of the material $(T \ll \tau)$, most of the pump energy is transferred to useful inversion $\left(E_{\mathrm{abs}, \mathrm{s}} \sim P_{\mathrm{abs}} T\right)$, without allowing the spontaneous emission process to relax the inverted ions. As the pump pulse width increases, the spontaneous emission process reduces the available inversion for amplification. At low repetition rates, the abovementioned formula for isolated pulse absorption, could be used in effectively estimating the overall absorption $\left(E_{\mathrm{abs}} \sim E_{\mathrm{abs}, s}\right)$. On the other hand, as the operating repetition rate of the regenerative amplifier $\left(f_{\text {rep }}\right)$ increases, the effective inversion is shared by multiple pulses, and one needs to calculate the amount of inversion left over from previous pump pulses as well. In such a case, the total available absorbed pump energy $\left(E_{\text {abs }}\right)$ for amplification is then calculated using

$$
E_{\mathrm{abs}}=E_{\mathrm{abs}, s}+\sum_{m=1}^{N}\left(E_{\mathrm{abs}, s}-E_{\mathrm{used}}\right) \operatorname{Exp}\left(-\frac{m}{\tau f_{\mathrm{rep}}}\right),
$$

where $E_{\text {used }}$ is the total used energy in the amplification process, which accounts for energy lost due to losses $\left(E_{\text {lost }}\right)$ and energy extracted from the amplifier by the earlier pulse $\left(E_{\text {out }}\right.$, which is the same as the output energy in a stable regen). Note that at higher repetition rates, the equation calls for an iterative solution, since the energy extracted from the system by earlier pulses (or output energy of the regenerative amplifier) determines the gain/inversion left for the circulating pulse.

For simplicity, in the simulations performed here, we assume $\mathrm{cw}$ pumping conditions, and the pump pulse width $(\mathrm{T})$ is then taken as $1 / f_{\text {rep }}$, where $f_{\text {rep }}$ is the repetition rate of the regenerative amplifier. Under this assumption, for higherrepetition-rate operation $\left(f_{\text {rep }} \gg 1 / \tau \sim 500 \mathrm{~Hz}\right)$, the pump pulses are much shorter than the fluorescence lifetime of the Yb:YLF gain medium, and clearly $\mathrm{cw}$ pumping does not provide any significant drawback. On the other hand, the advantages of pulsed pumping at low-repetition-rate operation is clear from Eq. (27); hence, the estimated performance at low repetition rates in this study could be improved upon pulsed pumping.

In order to solve for the evaluation of the pulse energy in the amplifier, we have applied Eq. (20) iteratively, with the output energy of the $n$th pass taken as the input energy of the $(n+1)$ th pass. In each pass, the variation in the stored energy in the upper laser level and the effect of cavity losses (L) are also included. Note also that the effective cavity losses $(\mathrm{L})$ include the passive losses $\left(L_{p}\right)$ of the optical elements, self-absorption losses $\left(L_{\mathrm{sa}}\right)$ of the $\mathrm{Yb}$ :YLF crystal, and additional losses observed under thermal load $\left(L_{\mathrm{ter}}\right.$, such as clipping of the beam by the PC aperture due to beam walk-off caused by one side cooling of the Yb:YLF crystal). For the $2 \mathrm{~cm}$ long $1 \% \mathrm{Yb}$-doped Yb:YLF crystal modeled in this work, the temperature variation of single-pass self-absorption losses at the central wavelength of $1016 \mathrm{~nm}$ could be estimated using (in the units of \%)

$L_{\mathrm{sa}, 1016}(T) \cong 0.034-0.001(T-77)+0.000025(T-77)^{2}$.

By investigating our earlier experimental data [15], we estimate a total loss level of around $10 \%$ for the $1016 \mathrm{~nm}$ Yb:YLF regenerative amplifier working under a total absorbed pump power load of $400 \mathrm{~W}$. Around $0.5 \%$ of this loss is estimated to be due to selfabsorption losses $\left(L_{\mathrm{sa}}\right)$ based on the thermal simulations of the system, as will be discussed in the next section.

\section{THERMO-MECHANICAL SIMULATIONS}

We start presentation of the simulation results with Fig. 6, which shows the estimated 3D temperature distribution within the $\mathrm{Yb}$ :YLF rod at an absorbed pump power of $400 \mathrm{~W}$. The calculation is performed for an assumed FTL of around 8.3\%, which corresponds to around $33 \mathrm{~W}$ of thermal load on the crystal. As we can see in Fig. 6, the temperature distribution inside the $\mathrm{Yb}: \mathrm{YLF}$ crystal is rather asymmetric. The asymmetry in the $y$ axis is due to the one-sided cooling of the crystal. On the other hand, one-sided pumping of the crystal and inhomogeneous distribution of the thermal load inside the crystal volume also contribute to the asymmetric thermal profile (creating asymmetry along the $z$ axis). The maximum temperature inside the crystal is estimated to be around $135 \mathrm{~K}$. On the other hand, the average temperature inside the crystal is only around $98 \mathrm{~K}$. Due to the un-doped caps on both sides, the crystal surfaces stay at reasonable temperatures, and the maximum temperatures on the front and back surfaces of the crystal are estimated to be $105 \mathrm{~K}$ and $96 \mathrm{~K}$, respectively.

For an accurate modeling of amplification/gain, the temperature of the crystal needs to be known. On the other hand, 

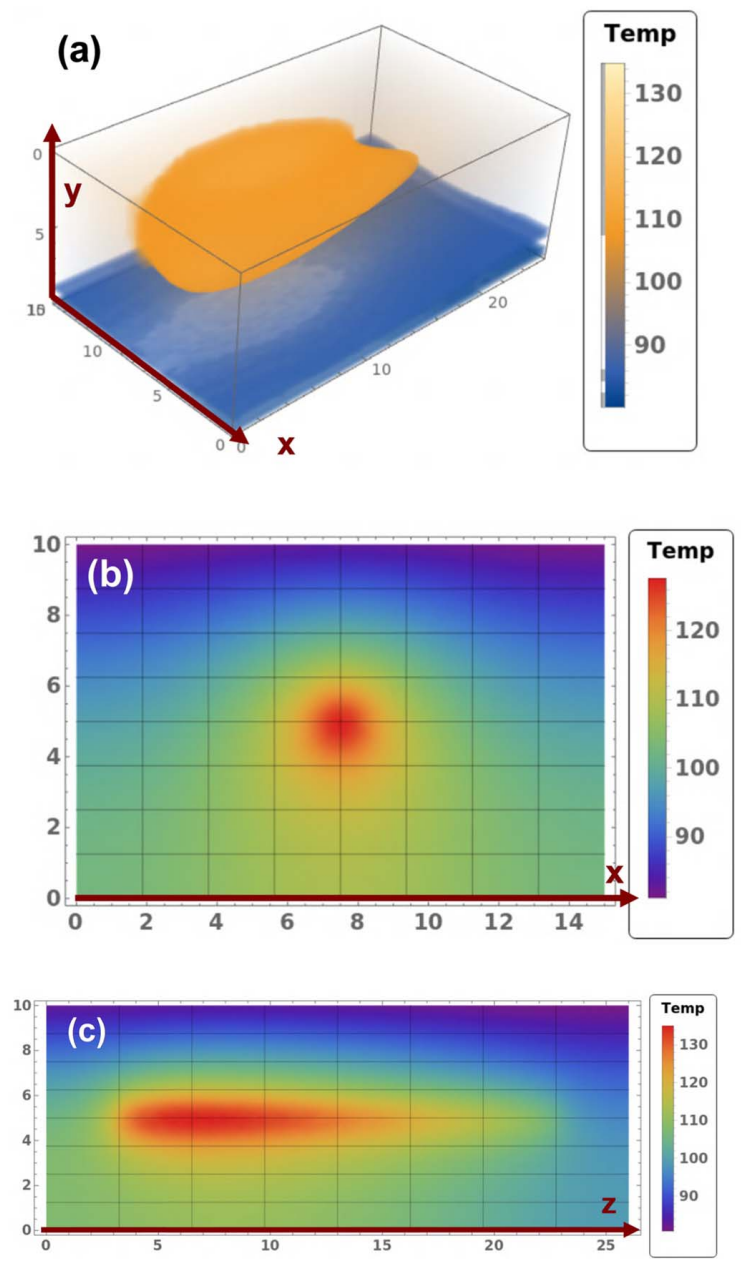

Fig. 6. Calculated temperature profile of the Yb:YLF crystal at an absorbed pump power of $400 \mathrm{~W}$. The pump beam waist has a size of $1 \mathrm{~mm}$ and is positioned at the center of the crystal. The dimensions are in $\mathrm{mm}$, and the temperatures are in K. (a) Full 3D profile of temperature; (b), (c) 2D temperature profiles at the planes of $(x, y, z=\mathrm{LL} / 2)$, and $(x, y=\mathrm{H} / 2, z)$, respectively.

a detailed 3D modeling for amplification is time consuming with current computational capabilities, and also is not really necessary for a reasonable estimation of amplification. Hence, in this work, for a practical estimation of spectroscopic properties, we have used the average temperature within the central region of the gain element as a first-order estimate for properties. The average temperature inside the seeded region of the gain element is calculated to be around $120 \mathrm{~K}$ for the $1016 \mathrm{~nm} \mathrm{Yb:YLF} \mathrm{crystal}$ under an absorbed pump load of $400 \mathrm{~W}$. Note that we have made several assumptions in thermal simulations including the value of the heat transfer coefficient at the boundary, the FTL and the value of thermal conductivity for 1\%-doped Yb:YLF. Hence, the temperature calculations we have made here should be considered as best effort estimates based on available data.

Another important issue to be addressed here is the estimation of thermal lensing of the Yb:YLF rod under thermal load. This information is useful not only for the successful design of regenerative amplifier cavities, but also for better understanding of limitations imposed by single-side cooling geometry. In our system, the seed beam travels along the $z$ axis; hence,

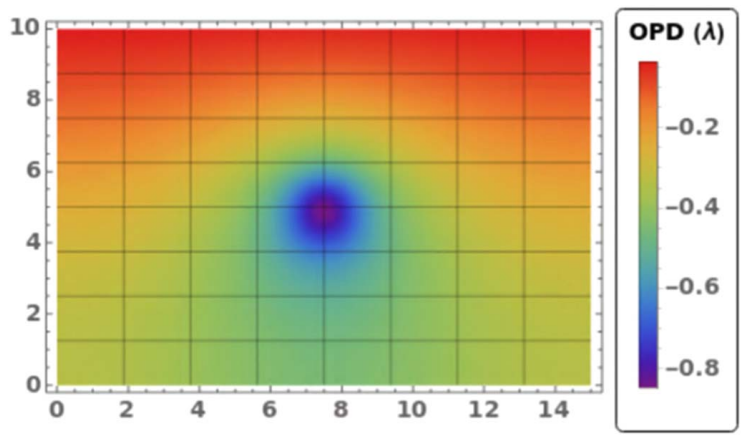

Fig. 7. Calculated variation of optical path difference (OPD) along the Yb:YLF crystal surface due to thermo-optic effect (variation of index of refraction with temperature). The dimensions are in $\mathrm{mm}$, and the OPD is specified in units of waves. The calculation was performed for a $1016 \mathrm{~nm} \mathrm{Yb:YLF} \mathrm{regenerative} \mathrm{amplifier} \mathrm{crystal,} \mathrm{using} \mathrm{a} \mathrm{pump}$ beam waist of $1 \mathrm{~mm}$ and an absorbed pump power of $400 \mathrm{~W}$.

we need to calculate the OPD that will be observed for a beam traveling through the $z$ axis. For that purpose, Fig. 7 shows the calculated OPD across the crystal cross section (width: $15 \mathrm{~mm}$, height: $10 \mathrm{~mm}$ ) due to the thermo-optic effect for a beam traveling along the $z$ axis. As we expected from earlier calculation of the temperature distribution inside the gain element [Fig. 6(b)], the OPD acquired due to the thermo-optic effect is quite asymmetric along the vertical axis due to the single-side cooling geometry applied [ $y$ axis in our geometry, shown in Fig. 3(b)]. The OPD due to $d n / d T$ is almost zero on the top part of the crystal, where the crystal temperature is relatively low, whereas an OPD value of up to $-0.8 \lambda$ is observed at the center of the crystal.

To understand the temperature-induced deformations, Fig. 8 shows the calculated surface bulging on the (a) front and (b) back surfaces of Yb:YLF gain media. Due to the un-doped cap sections on both ends, the estimated deformations are quite small (at the central region where the beam transverses). Moreover, as expected, the front surface deforms more than the back surface, due to the larger thermal load it receives. Note that the negative and positive values of the calculated deformations in Figs. 8(a) and 8(b) just indicate the direction of deformation (both surfaces expand with temperature).

At this point, it is beneficial to look at the total OPD acquired due to thermo-optic effects and surface deformations. For that purpose, we have chosen to present results along the $y$ axis in detail (Fig. 9), due to the aforementioned asymmetry problem in this axis. Fig. 9(a) shows the variation of calculated OPD due to thermo-optic effects along the $y$ axis, through the center of the crystal $(x=\mathrm{W} / 2)$. A quadratic fit to the OPD data results in an estimated thermal lens of around $-2 \mathrm{~m}$ focal length for the thermo-optic effect.

Figure 9(b) shows the variation of OPD due to surface bulging along the $y$ axis, passing through the center of the crystal $(x=\mathrm{W} / 2)$. As mentioned earlier, the un-doped cap sections help in this respect, and the estimated focal length of the thermal lens due to surface deformations is only around $12 \mathrm{~m}$. Finally, Fig. 9(c) shows the calculated total OPD due to thermo-optic and surface deformation effects, where the expected thermal lens has a focal length of around $-2.5 \mathrm{~m}$. The aspherical part 

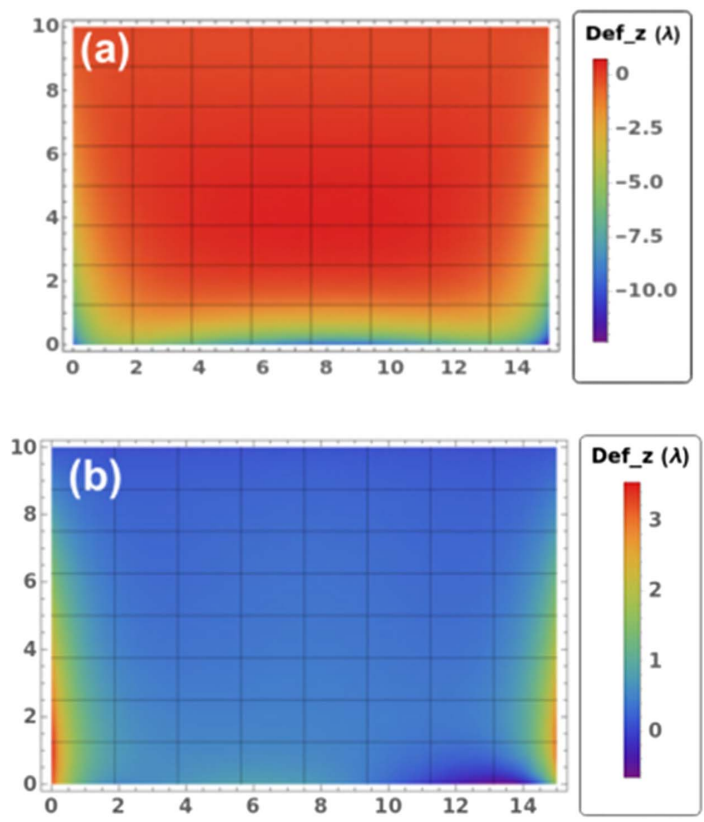

Fig. 8. Calculated temperature-induced deformation on the (a) front and (b) back surfaces of the Yb:YLF crystal. The dimensions are in $\mathrm{mm}$, and the path differences are specified in units of waves. The calculation was performed for a $1016 \mathrm{~nm}$ Yb:YLF regenerative amplifier crystal, using a pump beam waist of $1 \mathrm{~mm}$ and an absorbed pump power of $400 \mathrm{~W}$.

of the OPD curve is also shown in Fig. 9(c), and the estimated phase fluctuations in the central region (within $2 \mathrm{~mm}$ width) are within $\pm \lambda / 40$.

Note that the aspherical part of the OPD curve has a clear tilt component due to one-sided cooling of Yb:YLF crystal (an OPD difference of around $\lambda / 80$ for a $2 \mathrm{~mm}$ diameter beam). The estimated OPD accounts for a wedge angle of about $12 \mu \mathrm{rad}$ and an angular deviation of around $6 \mu \mathrm{rad}$. As a result, a seed beam passing though the YLF rod is expected to deviate around $20 \mu \mathrm{m}$ after $3 \mathrm{~m}$ of travel $(3 \mathrm{~m}$ is the modeled regenerative amplifier cavity length in this study). As we will see in the next section, a regenerative amplifier might require up to 100 round trips, especially for amplification at higher repetition rates, resulting in an overall beam walk-off as large as $2 \mathrm{~mm}$. It is clear that the hard aperture on the PC (6 $\mathrm{mm}$ in diameter) and the soft aperture on the Yb:YLF crystal ( $2 \mathrm{~mm}$ in diameter) will work against this shift. Effectively, the process will result in a reduction in mode-matching efficiency and in an overall increase in effective cavity losses.

In a similar manner, for the symmetric $x$ axis, we have estimated thermo-optic, deformation, and total lensing as $-2.4 \mathrm{~m}$, $125 \mathrm{~m}$, and again roughly as $-2.5 \mathrm{~m}$, respectively. We see from our simple analysis then that the thermo-optic effect is dominant over surface deformations in the end-capped Yb:YLF crystal used in this study, and due to the negative $d n / d T$ coefficient, a negative thermal lens with a focal length of roughly around $-2.5 \mathrm{~m}$ is estimated from the $\mathrm{Yb}$ :YLF crystal under the aforementioned conditions. On the other hand, in earlier experimental work, a positive and weak thermal lens $(\sim+5-10 \mathrm{~m})$ is reported from a similar Yb:YLF system (a $2 \mathrm{~cm}$ long 0.5\%-doped Yb:YLF sample was used in the experiments [15]). This discrepancy calls for a detailed investigation of
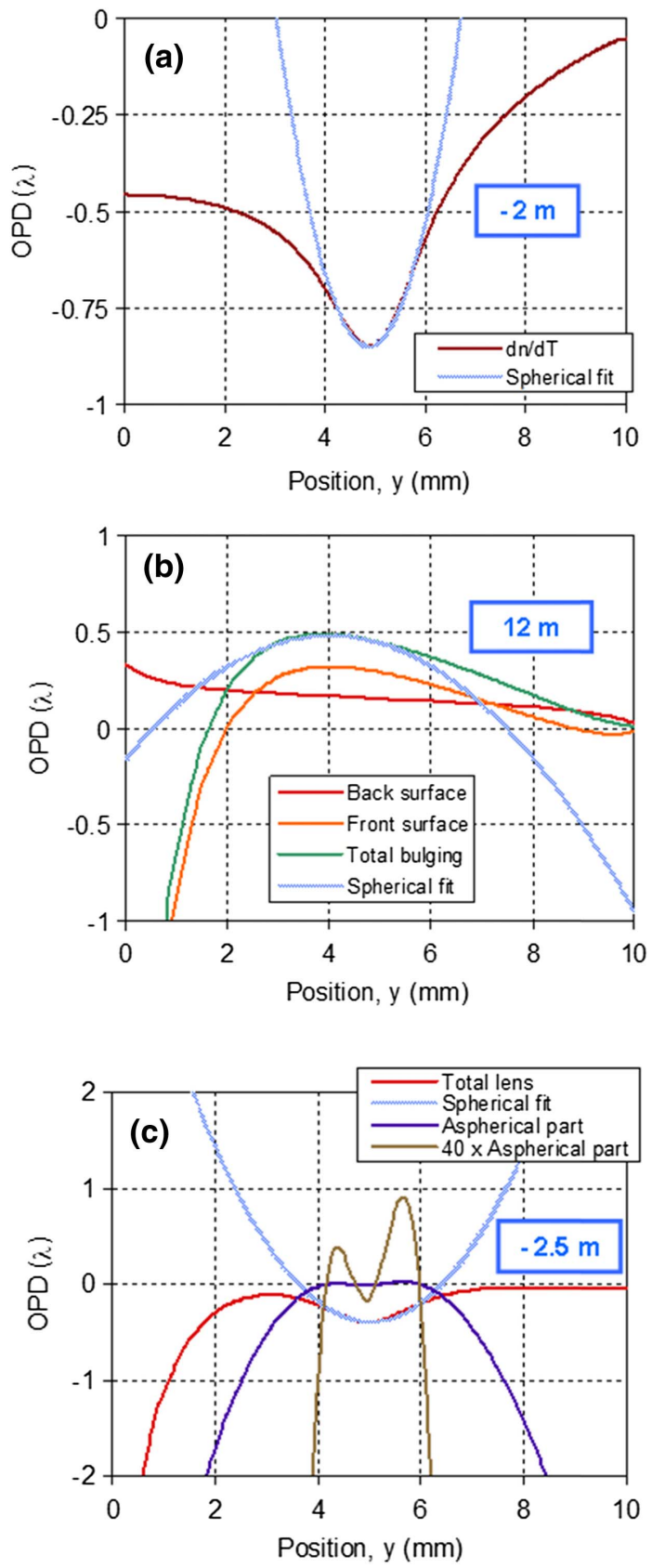

Fig. 9. (a) Calculated OPD due to the variation of refractive index with temperature (thermo-optic effect) in the $y$ axis at $x=\mathrm{W} / 2$. The spherical component has a focal length of around $-2 \mathrm{~m}$. (b) Calculated optical path difference due to deformation/bulging of the front and back surfaces of the Yb:YLF rod in the $y$ axis. The total OPD due to bulging is also shown. The spherical component of the deformation lens has a focal length of around $12 \mathrm{~m}$. (c) Estimated variation of total (bulging $+d n / d T$ ) OPD along the $y$ axis. The spherical component of the total lens has an estimated focal length of around $-2.5 \mathrm{~m}$. The remaining aspherical component and its 40 times magnified version are also shown. The calculation was performed for a $2 \mathrm{~cm}$ long $1 \%$ Yb-doped Yb:YLF regenerative amplifier crystal containing $3 \mathrm{~mm}$ un-doped wedges on both surfaces. We have assumed a pump beam waist of $1 \mathrm{~mm}$ and an absorbed pump power of $400 \mathrm{~W}$.

thermal lensing in Yb:YLF. The observed difference might be due to the presence of a relatively strong population/electronic lensing in Yb:YLF [42], and/or due to the contribution of the 
photo-elastic effect, as shown for the case of room-temperature Nd:YLF [44]. For our work here, we are focused on an accurate estimation of amplification performance, and a detailed knowledge of thermal lensing is not required at this stage, but would be necessary for experimentalists for optimization of the regenerative amplifier cavity design. On the positive side, the experimentally observed lens in YLF is relatively small compared to other systems such as $\mathrm{Yb}: \mathrm{YAG}$, easing the design of amplifier systems in rod geometry.

\section{ESTIMATED REGENERATIVE AMPLIFIER PERFORMANCE}

In Section 4.A, we first discuss regenerative amplifier results using a single Yb:YLF crystal as a gain element, where we have obtained simulation results that are in good agreement with our recent experimental work [15]. In Section 4.B, we describe how the performance can be further improved using a dual-rod geometry.

\section{A. Single-Crystal Regen Amplification Results}

We start this section with Fig. 10, which shows the estimated regenerative amplifier performance of Yb:YLF at several different repetition rates between $1 \mathrm{~Hz}$ and $5 \mathrm{kHz}$. Specifically, Fig. 10 shows the increase in pulse energy as a function of round trips, for a seed energy of $10 \mathrm{~nJ}$. The simulation parameters are summarized in the figure caption. Due to laser-induced damage, we limit the safe operation limit of the amplifier to $35 \mathrm{~mJ}$, which is marked with the gray solid horizontal line. This corresponds to a peak operation fluence of around $2.25 \mathrm{~J} / \mathrm{cm}^{2}$, a value considerably lower than the estimated damage fluence $\left(\sim 9 \mathrm{~J} / \mathrm{cm}^{2}\right)$ for the stretched 2 ns pulses. As can be seen, at low repetition rates, the amplifier easily reaches the $35 \mathrm{~mJ}$ energy level. Once the repetition rate exceeds $1 / \tau(\sim 500 \mathrm{~Hz})$, the accumulated inversion is shared between pulses, which effectively decreases the effective net gain per pulse, and increases the number of round trips required to reach $35 \mathrm{~mJ}$ energy. As an example, while it takes only around 43 round trips at $1 \mathrm{~Hz}, 67$ round trips are required to reach $35 \mathrm{~mJ}$ energy at $2 \mathrm{kHz}$ repetition rate. As the repetition rate increases further (frep $>2 \mathrm{kHz})$ ), the acquired pulse energies become limited by the extractable average output power from the system. As an example, at a repetition rate of $5 \mathrm{kHz}$, only $17 \mathrm{~mJ}$ are extractable from the system after 96 round trips. As a positive point, the estimated B-integral of the system is still around 0.7 even for this large number of round trips.

As another point, looking at the slope of the curve in Fig. 10, one can ask whether higher energies could be extracted at higher repetition rates by increasing the number of round trips in the regenerative amplifier. To answer this question, Fig. 11 shows the estimated performance of the regenerative amplifier at $5 \mathrm{kHz}$ repetition rate for several different output energy levels. The graph shows that it is possible to reach $11.3 \mathrm{~mJ}$ energy at $5 \mathrm{kHz}$ in just 55 round trips. On the other hand, as we can see, as the desired output energy is increased, the required number of round trips inflates in an accelerating trend. Basically, the system is having difficulty in providing enough inversion/gain for the extra energy that is desired. At some point, once the output energy reaches the extraction limit $(17 \mathrm{~mJ}$ of energy at $5 \mathrm{kHz}$

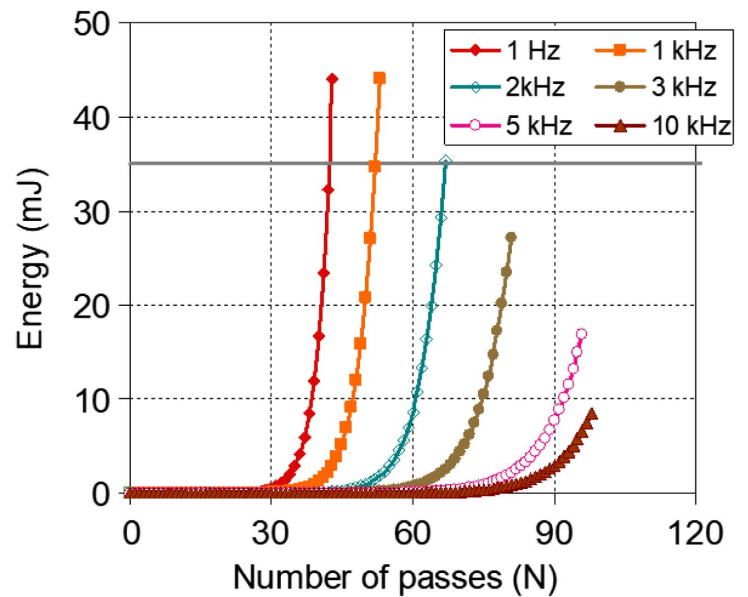

Fig. 10. Calculated variation of single-crystal $1016 \mathrm{~nm}$ Yb:YLF cryogenic regenerative amplifier output energy as a function of number of round trips at different repetition rates between $1 \mathrm{~Hz}$ and $5 \mathrm{kHz}$. Due to LIDT, the obtainable pulse energy is limited to around $35 \mathrm{~mJ}$ (shown with the horizontal gray line). Simulation parameters: $w_{p}=1 \mathrm{~mm}, \mathrm{MMF} \cong 70 \%$, loss $\cong 10 \%, \tau_{s}=2 \mathrm{~ns}, P_{\mathrm{abs}}=350 \mathrm{~W}+$ $1 / 2 P_{\text {out }}$.

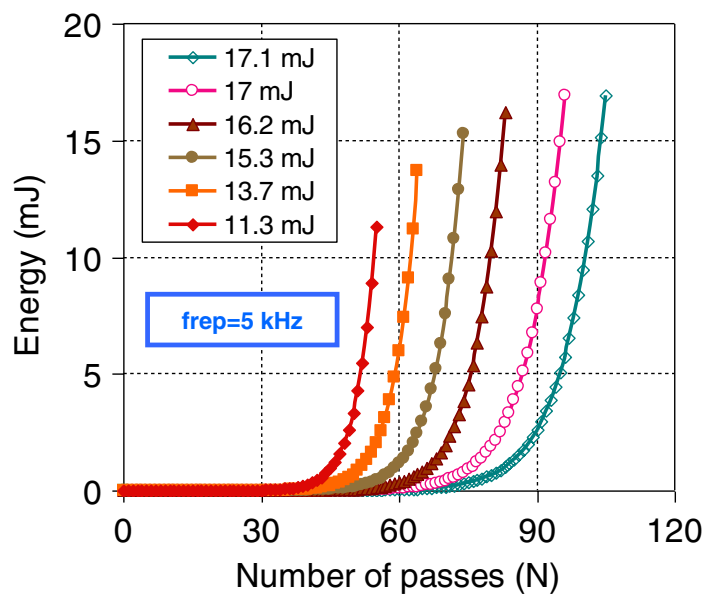

Fig. 11. Calculated variation of single-crystal $1016 \mathrm{~nm}$ Yb:YLF cryogenic regenerative amplifier performance at $5 \mathrm{kHz}$ repetition rate at different output energy levels. The maximum extractable output energy is limited to around $17 \mathrm{~mJ}$ in this configuration (corresponds to $85 \mathrm{~W}$ average power). Simulation parameters: $w_{p}=1 \mathrm{~mm}$, $\mathrm{MMF} \cong 70 \%$, loss $\cong 10 \%, \tau_{s}=2 \mathrm{~ns}, P_{\mathrm{abs}}=350 \mathrm{~W}+\frac{1 / 2}{2} P_{\text {out }}$.

for this particular situation), additional round trips do not provide much benefit in terms of output energy. As we can see in Fig. 15, almost no benefit is obtained in terms of extractable energy when the round trip increases from 96 to 105. Basically, this shows that the effective gain per pulse reduces to the level of cavity losses, and another round trip in the cavity provides no extra amplification.

To elaborate on this issue, Fig. 12 shows extractable energy levels from the single-crystal $1016 \mathrm{~nm}$ Yb:YLF regenerative amplifier at different repetition rates. As mentioned above, for the assumed stretched pulse width ( $2 \mathrm{~ns})$, we limit the pulse energies to the $35 \mathrm{~mJ}$ level (and peak fluence to $2.25 \mathrm{~J} / \mathrm{cm}^{2}$ ) for 


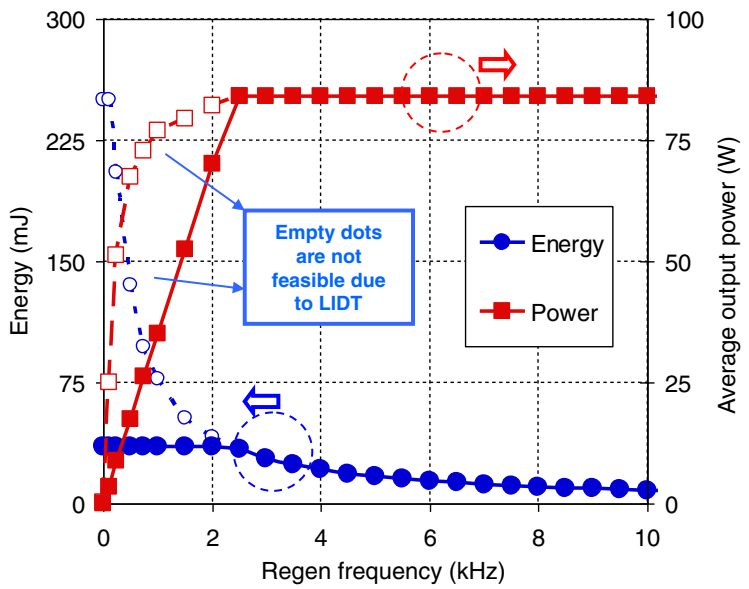

Fig. 12. Estimated performance of single-crystal $1016 \mathrm{~nm}$ Yb:YLF cryogenic regenerative amplifier as a function of repetition rate. Due to LIDT, the obtainable pulses are limited to around $35 \mathrm{~mJ}$ at low repetition rates. Empty spots with energies up to $250 \mathrm{~mJ}$ are not feasible, but are shown on purpose to illustrate the extraction limits of the system for a cw seed. At higher repetition rates, obtainable energies are limited by the achievable average power level $(\sim 85 \mathrm{~W})$ from the system. Simulation parameters: $w_{p}=1 \mathrm{~mm}, \mathrm{MMF} \cong 70 \%$, loss $\cong 10 \%$, $\tau_{s}=2 \mathrm{~ns}, P_{\mathrm{abs}}=350 \mathrm{~W}+1 / 2 P_{\mathrm{out}}$.

long-term damage-free operation. On the other hand, Fig. 12 shows that, ignoring LIDT, the system actually allows output energies up to $250 \mathrm{~mJ}$ at low repetition rates (shown by empty dots). Hence, it is clear that the stretched pulse width of the pulses will determine the obtainable energy levels at low repetition rates: which are roughly around $35 \mathrm{~mJ}$ level with $2 \mathrm{~ns}$ pulses, $50 \mathrm{~mJ}$ level for $4 \mathrm{~ns}$ pulses, $24 \mathrm{~mJ}$ level for $1 \mathrm{~ns}$ pulses, etc. At high repetition rates, as discussed above, the system is saturated at an average power of around $85 \mathrm{~W}$, which limits the output energies to around $17 \mathrm{~mJ}$ at $5 \mathrm{kHz}$, and to $8.5 \mathrm{~mJ}$ at $10 \mathrm{kHz}$. In short, we see that pulse energies are limited by LIDT at low repetition rates, and by the extraction efficiency and achievable overall inversion of the system at high repetition rates.

At this point, it is instructive to investigate the effect of system parameters on performance, especially the amplifier performance at higher repetition rates, where the extraction efficiency is a limiting factor. To start with, Fig. 13(a) shows the effect of single-pass cavity losses $(\mathrm{L})$ in regenerative amplifier performance. As mentioned above, in the analysis, we assume an effective cavity loss level of $10 \%$, which was the estimated loss level in our recent experimental work [15]. As we can see in Fig. 13(a), the estimated performance at higher repetition rates is a strong function of losses. As an example, the achievable energy level at $10 \mathrm{kHz}$ increases from around $8.5 \mathrm{~mJ}$ to $12.5 \mathrm{~mJ}$ level, if one can minimize the cavity losses from around $10 \%$ to $5 \%$, which might be achievable, if one optimizes the passive losses of all the elements in the cavity [15].

Another important parameter that has quite an impact on amplifier performance is the absorbed pump power $\left(P_{\mathrm{abs}}\right)$. Figure 13(b) shows the effect of pump power on amplifier performance. In the simulations above, we assumed that thermal effects limit the safe operation to an absorbed pump power level of $350 \mathrm{~W}+1 / 2 P_{\text {out }}$ (a rough experimental trend we
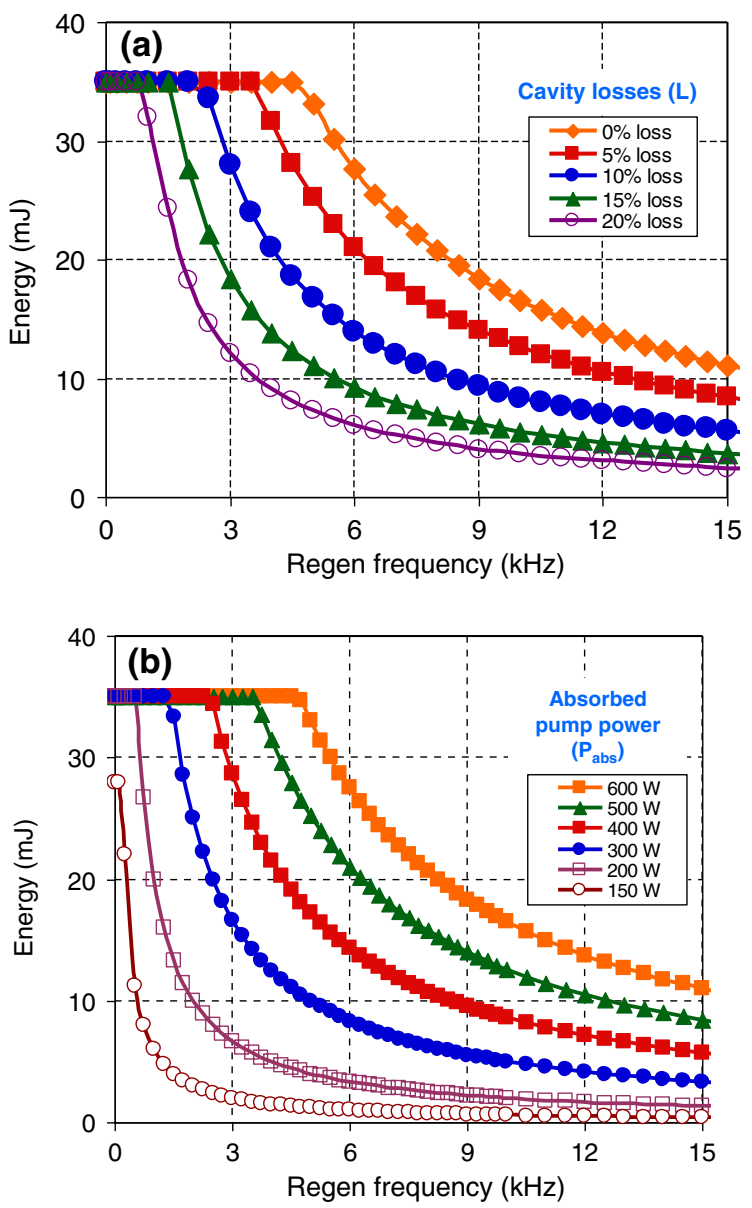

Fig. 13. Estimated effect of (a) cavity losses (L) and (b) absorbed pump power level $\left(P_{\mathrm{abs}}\right)$ on the single-crystal cryogenic $1016 \mathrm{~nm}$ Yb:YLF regenerative amplifier. Simulation parameters: $w_{p}=1 \mathrm{~mm}$, $\mathrm{MMF} \cong 70 \%$.

observed in our detailed lasing experiments in Ref. [1]). Hence, for the average output power of $85 \mathrm{~W}$, we assume an absorbed pump power of $392.5 \mathrm{~W}$ in our analysis above. As can be seen in Fig. 13(b), if one finds ways to minimize the thermal effects, this could boost the amplifier performance at higher repetition rates. As an example, assuming a $600 \mathrm{~W}$ absorbed pump power level, the estimated performance at $10 \mathrm{kHz}$ improves from $8.5 \mathrm{~mJ}$ to $16.5 \mathrm{~mJ}$ level. On the other hand, as we have discussed in the thermo-mechanical simulation section, application of pump powers above $400-500 \mathrm{~W}$ might be very challenging without significantly improving the heat transfer ability of the liquid nitrogen boundary [30,31]. Furthermore, the thermally induced deformations (thermal lensing) could also create stability problems in the regen cavity, resulting in undesired perturbation in the regen beam profile at these pump power levels.

\section{B. Dual-Crystal Regen Amplification Results}

As we have seen above, we expect the thermal effects to limit the extractable average powers to around $85 \mathrm{~W}$ in single crystal $\mathrm{Yb}: \mathrm{YLF}$ rod amplifiers operating at around $1016 \mathrm{~nm}$. In this section, we investigate possible improvements that could be 


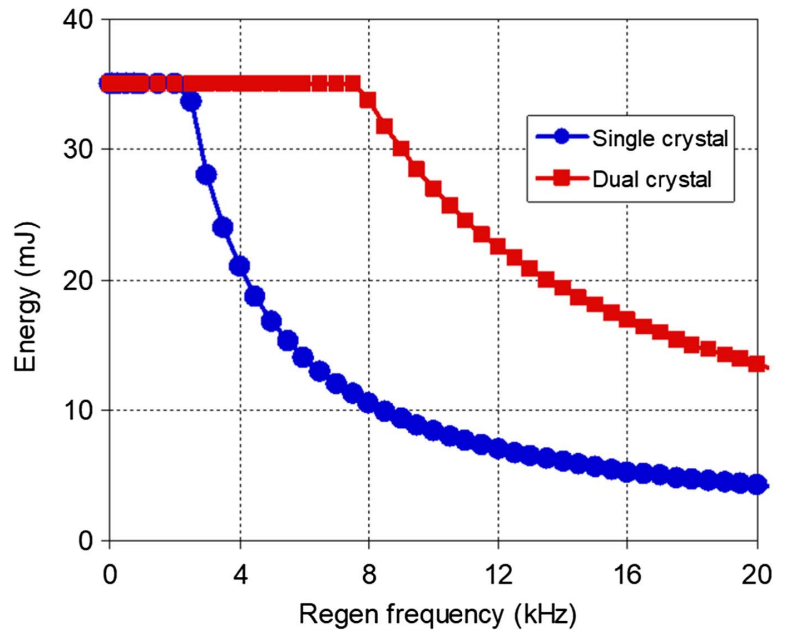

Fig. 14. Compared performance of dual-crystal and single-crystal $1016 \mathrm{~nm}$ cryogenic Yb:YLF regenerative amplifier. Simulation parameters: $w_{p}=1 \mathrm{~mm}, \mathrm{MMF} \cong 70 \%$, loss $\cong 10 \%, \tau_{s}=2 \mathrm{~ns}$.

achieved by employing two Yb:YLF crystals in the cavity, as have been applied to other systems earlier [50-52]. For this purpose, Fig. 14 compares the estimated performance of singleand dual-crystal $\mathrm{Yb}: \mathrm{YLF}$ regenerative amplifiers. In the analysis, we assume that the dual-crystal geometry could handle a total absorbed pump power of $700 \mathrm{~W}+1 / 2 P_{\text {out }}$. All the other simulation parameters are kept constant. As we can see, the dual-rod geometry significantly increases the net gain of the system. As a result, the extractable average power from the system increases from around $85 \mathrm{~W}$ to $270 \mathrm{~W}$, which increases the extractable energies from $8.5 \mathrm{~mJ}$ to $27 \mathrm{~mJ}$ at $10 \mathrm{kHz}$. Besides improving the extractable energies at high repetition rates, the dual-rod geometry also decreases the required number of round trips significantly. As an example, the required number of round trips to reach $35 \mathrm{~mJ}$ pulses decreases from 57 to only 22 at $1 \mathrm{kHz}$ repetition rate.

So far in our analysis, we have assumed a spot size $\left(w_{0}\right)$ of $1 \mathrm{~mm}$. Here, we want to elaborate on the estimated effect of pump/cavity spot size on regenerative amplifier performance. Fig. 15 shows the variation of extractable energies with repetition rate in dual-rod geometry at several different spot sizes ranging from $0.75 \mathrm{~mm}$ to $2 \mathrm{~mm}$. As one can see, the $1 \mathrm{~mm}$ spot size provides the optimum performance at high repetition rates, since it allows good mode matching between the laser/regen and pump modes for the specific $2 \mathrm{~cm}$ long Yb:YLF crystal (which is also experimentally confirmed in Refs. [1,15]). For tighter spot sizes, amplifier performance deteriorates due to the decreasing mode-matching efficiency. On the other hand, for large spot sizes, the decreasing gain limits the achievable output power levels. On the low-repetition-rate side, a larger spot size has advantages, since it enables handling of higher pulse energies. As an example, increasing the spot size from $1 \mathrm{~mm}$ to $2 \mathrm{~mm}$, one can increase the safe operation point of the $\mathrm{Yb}$ :YLF regenerative amplifier from around $35 \mathrm{~mJ}$ to $140 \mathrm{~mJ}$. As a disadvantage, a larger beam size would: (i) reduce gain and increase sensitivity of the cavity, and (ii) require larger optical elements, including larger-sized Yb:YLF rods, and PCs, which increases the cost of

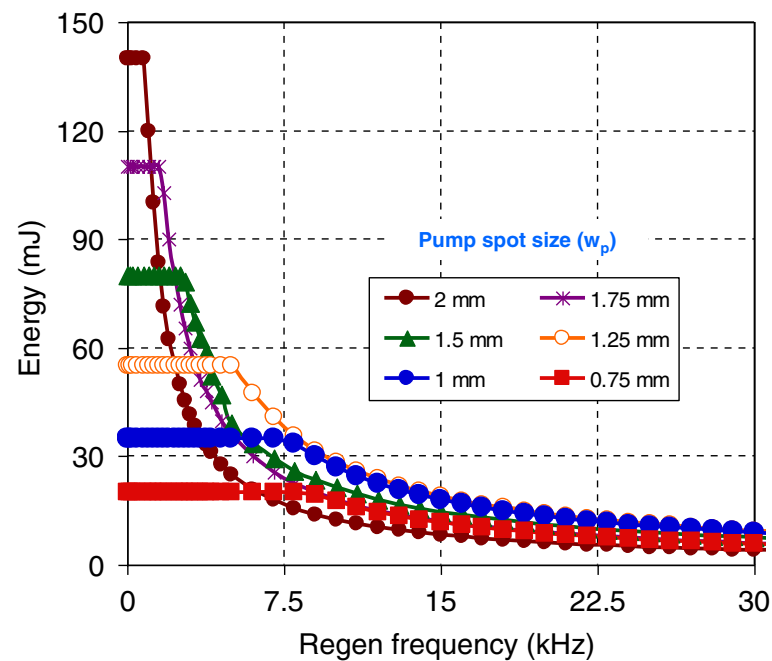

Fig. 15. Estimated effect of pump spot size $\left(w_{p}\right)$ on the dual-crystal cryogenic $1016 \mathrm{~nm}$ Yb:YLF regenerative amplifier. Simulation parameters: $\mathrm{MMF} \cong 70 \%$, loss $\cong 10 \%, \tau_{s}=2 \mathrm{~ns}, P_{\text {abs }}=700 \mathrm{~W}+1 / 2 P_{\text {out }}$.

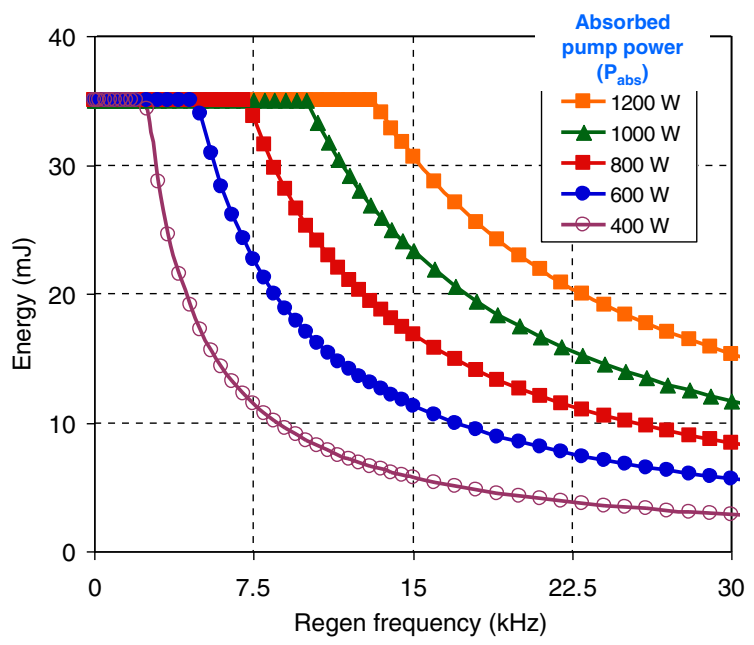

Fig. 16. Estimated effect of absorbed pump power $\left(P_{\mathrm{abs}}\right)$ on the dual-crystal cryogenic $1016 \mathrm{~nm}$ Yb:YLF regenerative amplifier. Simulation parameters: $w_{p}=1 \mathrm{~mm}, \quad \mathrm{MMF} \cong 70 \%$, loss $\cong 10 \%$, $\tau_{s}=2 \mathrm{~ns}$.

the system. Moreover, design/implementation of cavities with larger spot sizes is known to be more challenging.

In closing this section, we will discuss the estimated effect of the total absorbed pump power on the dual-crystal regenerative amplifier. In our analysis in Fig. 15, we assume an absorbed pump power level of $700 \mathrm{~W}+1 / 2 P_{\text {out }}$, which corresponds to an absorbed pump power level of about $835 \mathrm{~W}$. As can be seen in Fig. 1(b), in dual-rod geometry, the Yb:YLF crystals need to be placed at a reasonable distance from each other to enable good mode matching. On the other hand, this in turn might create some thermal crosstalk between the crystals that could limit the total absorbed pump power level. Moreover, in dual-rod geometry, the thermal lens of each crystal will add up, and the overall effective thermal lens of the cavity will be larger, which could create challenges in cavity design. To elaborate on this 


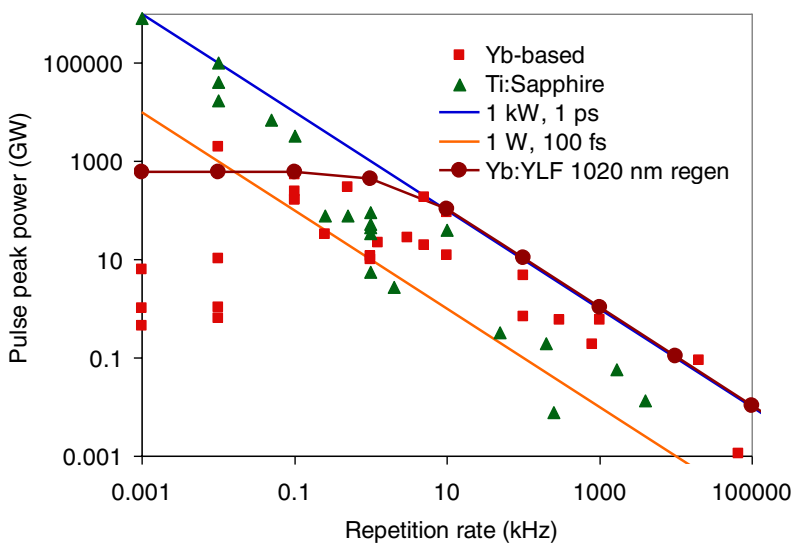

Fig. 17. Comparison of the estimated performance of the Yb:YLF regenerative amplifier with literature in terms of peak power that could be achieved as a function of repetition rate.

issue, Fig. 16 shows the variation of estimated performance with absorbed pump power level. In our earlier experimental work, we showed that a single rod could be safely pumped by almost $400 \mathrm{~W}$ pump power [1]. Hence, we believe that ideally, the dual-rod geometry should handle $800 \mathrm{~W}$ of absorbed pump power. In the worst case, we expect the system to easily handle $600 \mathrm{~W}$ average pump power. Note that at an absorbed pump power of $600 \mathrm{~W}$, the system is estimated to still produce $170 \mathrm{~W}$ of average output power, which corresponds to a pulse energy of $17 \mathrm{~mJ}$ at $10 \mathrm{kHz}$.

\section{CONCLUSION}

In conclusion, we have performed a comprehensive numerical investigation of cryogenic Yb:YLF regenerative amplifier systems. The simulations have shown that a single-stage $\mathrm{Yb}$ :YLF regenerative amplifier employing dual Yb:YLF rods could potentially provide sub-250 fs pulses with an average power up to $270 \mathrm{~W}$ and peak power up to $600 \mathrm{GW}$. The system allows operation of the amplifier in either high-energy mode $(>100 \mathrm{~mJ}$ at $1 \mathrm{kHz})$, or high-average-power mode $(>25 \mathrm{~mJ}$ at $10 \mathrm{kHz}$, with $>250 \mathrm{~W}$ ). Figure 17 shows that the expected performance of the Yb:YLF regenerative amplifier in terms of peak power can already compete with other Yb-based or Ti:-sapphire-based systems in the literature. Moreover, this system could be used as a front end for further Yb:YLF amplifier systems, which could scale the performance of Yb:YLF amplifiers further [23]. The discussed $\mathrm{Yb}$ :YLF-based amplifier systems can be very useful for applications such as pumping of high-energy and averagepower optical parametric amplifiers, terahertz generation, spectral broadening and compression of high-energy pulses, and ultrafast $\mathrm{x}$-ray generation [53-55].

Funding. European Research Council (609920).

Acknowledgment. U. D. acknowledges support from the BAGEP Award of the Bilim Akademisi.

Disclosures. The authors declare no conflicts of interest.

\section{REFERENCES}

1. U. Demirbas, H. Cankaya, J. Thesinga, F. X. Kartner, and M. Pergament, "Efficient, diode-pumped, high-power $>300 \mathrm{~W}$ ) cryogenic Yb:YLF laser with broad-tunability (995-1020.5 nm): investigation of E//a-axis for lasing," Opt. Express 27, 36562-36579 (2019).

2. X. Delen, Y. Zaouter, I. Martial, N. Aubry, J. Didierjean, C. Honninger, E. Mottay, F. Balembois, and P. Georges, "Yb:YAG single crystal fiber power amplifier for femtosecond sources," Opt. Lett. 38, 109-111 (2013).

3. A. Giesen, H. Hugel, A. Voss, K. Wittig, U. Brauch, and H. Opower, "Scalable concept for diode-pumped high-power solid-state lasers," Appl. Phys. B 58, 365-372 (1994).

4. C. J. Saraceno, "Mode-locked thin-disk lasers and their potential application for high-power terahertz generation," J. Opt. 20, 4 (2018).

5. A. Giesen and J. Speiser, "Fifteen years of work on thin-disk lasers: results and scaling laws," IEEE J. Sel. Top. Quantum Electron. 13 598-609 (2007).

6. J. Brons, V. Pervak, D. Bauer, D. Sutter, O. Pronin, and F. Krausz, "Powerful 100-fs-scale Kerr-lens mode-locked thin-disk oscillator," Opt. Lett. 41, 3567-3570 (2016).

7. T. Nubbemeyer, M. Kaumanns, M. Ueffing, M. Gorjan, A. Alismail, H. Fattahi, J. Brons, O. Pronin, H. G. Barros, Z. Major, T. Metzger, D. Sutter, and F. Krausz, " $1 \mathrm{~kW}, 200 \mathrm{~mJ}$ picosecond thin-disk laser system," Opt. Lett. 42, 1381-1384 (2017).

8. L. E. Zapata, H. Lin, A. L. Calendron, H. Cankaya, M. Hemmer, F. Reichert, W. R. Huang, E. Granados, K. H. Hong, and F. X. Kartner, "Cryogenic Yb:YAG composite-thin-disk for high energy and average power amplifiers," Opt. Lett. 40, 2610-2613 (2015).

9. L. E. Zapata, F. Reichert, M. Hemmer, and F. X. Kartner, " 250 W average power, $100 \mathrm{kHz}$ repetition rate cryogenic $\mathrm{Yb}$ :YAG amplifier for OPCPA pumping," Opt. Lett. 41, 492-495 (2016).

10. J. Kawanaka, K. Yamakawa, H. Nishioka, and K. Ueda, "Improved high-field laser characteristics of a diode-pumped Yb:LiYF4 crystal at low temperature," Opt. Express 10, 455-460 (2002).

11. D. E. Miller, J. R. Ochoa, and T. Y. Fan, "Cryogenically cooled, 149 W, Q-switched, Yb:LiYF4 laser," Opt. Lett. 38, 4260-4261 (2013).

12. A. Bensalah, Y. Guyot, M. Ito, A. Brenier, H. Sato, T. Fukuda, and G. Boulon, "Growth of $\mathrm{Yb}^{3+}$-doped $\mathrm{YLiF}_{4}$ laser crystal by the Czochralski method. Attempt of $\mathrm{Yb}^{3+}$ energy level assignment and estimation of the laser potentiality," Opt. Mater. 26, 375-383 (2004).

13. A. Sugiyama, M. Katsurayama, Y. Anzai, and T. Tsuboi, "Spectroscopic properties of Yb doped YLF grown by a vertical Bridgman method," J. Alloys Comp. 408, 780-783 (2006).

14. U. Demirbas, J. Thesinga, H. Cankaya, M. Kellert, F. X. Kartner, and M. Pergament, "High-power passively mode-locked cryogenic Yb:YLF laser," Opt. Lett. 45, 2050-2053 (2020).

15. U. Demirbas, H. Cankaya, Y. Hua, J. Thesinga, M. Pergament, and F. X. Kaertner, "20-mJ, sub-ps pulses at up to $70 \mathrm{~W}$ average power from a cryogenic Yb:YLF regenerative amplifier," Opt. Express 28, 2466-2479 (2020).

16. J. Kawanaka, S. Tokita, H. Nishioka, M. Fujita, K. Yamakawa, K. Ueda, and Y. Izawa, "Dramatically improved laser characteristics of diode-pumped Yb-doped materials at low temperature," Laser Phys. 15, 1306-1312 (2005).

17. M. Vannini, G. Toci, D. Alderighi, D. Parisi, F. Cornacchia, and M. Tonelli, "High efficiency room temperature laser emission in heavily doped Yb : YLF," Opt. Express 15, 7994-8002 (2007).

18. N. Uehara, K. Ueda, and Y. Kubota, "Spectroscopic measurements of a high-concentration $\mathrm{Yb}^{3+}: \mathrm{LiYF}_{4}$ crystal," Jpn. J. Appl. Phys. 35, L499-L501 (1996).

19. J. Kawanaka, K. Yamakawa, H. Nishioka, and K. Ueda, "30-mJ, diode-pumped, chirped-pulse Yb : YLF regenerative amplifier," Opt. Lett. 28, 2121-2123 (2003).

20. D. Rand, D. Miller, D. J. Ripin, and T. Y. Fan, "Cryogenic $\mathrm{Yb}^{3+}$-doped materials for pulsed solid-state laser applications [Invited]," Opt. Mater. Express 1, 434-450 (2011).

21. D. E. Miller, L. E. Zapata, D. J. Ripin, and T. Y. Fan, "Sub-picosecond pulses at $100 \mathrm{~W}$ average power from a Yb:YLF chirped-pulse amplification system," Opt. Lett. 37, 2700-2702 (2012). 
22. J. Manni, D. Harris, and T. Y. Fan, "High-gain (43 dB), high-power $(40 \mathrm{~W})$, highly efficient multipass amplifier at $995 \mathrm{~nm}$ in Yb:LiYF 4 ," Opt. Commun. 417, 54-56 (2018).

23. H. Cankaya, U. Demirbas, Y. Hua, M. Hemmer, L. E. Zapata, M. Pergament, and F. X. Kärtner, "190-mJ cryogenically-cooled Yb:YLF amplifier system at $1019.7 \mathrm{~nm}, "$ OSA Continuum 2, 3547-3553 (2019).

24. N. Hodgson and A. Caprara, "Calculation of temperature profiles in solid-state laser disks mounted on multi-layered heat spreaders using Hankel transforms," Appl. Opt. 55, 10146-10157 (2016).

25. C. J. M. Lasance, "How to estimate heat spreading effects in practice," J. Electron. Packag. 132, 031004 (2010).

26. Y. Hua, W. Liu, M. Hemmer, L. E. Zapata, G. J. Zhou, D. N. Schimpf, T. Eidam, J. Limpert, A. Tunnermann, F. X. Kartner, and G. Q. Chang, "87-W 1018-nm Yb-fiber ultrafast seeding source for cryogenic $\mathrm{Yb}$ : yttrium lithium fluoride amplifier," Opt. Lett. 43, 1686-1689 (2018).

27. M. Kida, Y. Kikuchi, O. Takahashi, and I. Michiyoshi, "Pool-boiling heat-transfer in liquid-nitrogen," J. Nucl. Sci. Technol. 18, 501-513 (1981).

28. K. Bouazaoui, R. Agounoun, K. Sbai, A. Zoubir, I. Kadiri, M. Rahmoune, and R. Saadani, "Experimental and numerical study of pool boiling heat transfer of liquid nitrogen LN2: application to the brass ribbon cooling in horizontal position," Int. J. Mech. Mechatronics Eng. 17, 74-82 (2017).

29. B. Baudouy, "Heat transfer and cooling techniques at low temperature," arXiv: 1501.07153 (2015).

30. H. Hu, C. Xu, Y. Zhao, K. J. Ziegler, and J. N. Chung, "Boiling and quenching heat transfer advancement by nanoscale surface modification," Sci. Rep. 7, 1 (2017).

31. C. Kruse, T. Anderson, C. Wilson, C. Zuhlke, D. Alexander, G. Gogos, and S. Ndao, "Extraordinary shifts of the Leidenfrost temperature from multiscale micro/nanostructured surfaces," Langmuir 29, 9798-9806 (2013).

32. O. Slezak, A. Lucianetti, M. Divoky, M. Sawicka, and T. Mocek, "Optimization of wavefront distortions and thermal-stress induced birefringence in a cryogenically-cooled multislab laser amplifier," IEEE J. Quantum Electron. 49, 960-966 (2013).

33. R. L. Aggarwal, D. J. Ripin, J. R. Ochoa, and T. Y. Fan, "Measurement of thermo-optic properties of $\mathrm{Y}_{3} \mathrm{Al}_{5} \mathrm{O}_{12}, \mathrm{Lu}_{3} \mathrm{Al}_{5} \mathrm{O}_{12}, \mathrm{YAIO}_{3}$, LiYF 4 , $\mathrm{LiLuF}_{4}, \mathrm{BaY}_{2} \mathrm{~F}_{8}, \mathrm{KGd}\left(\mathrm{WO}_{4}\right)_{2}$, and $\mathrm{KY}\left(\mathrm{WO}_{4}\right)_{2}$ laser crystals in the 80-300 K temperature range," J. Appl. Phys. 98, 103514 (2005).

34. R. W. Powell, M. J. Woodman, and R. P. Tye, "Thermal conductivity and electrical resistivity of indium," Philos. Mag. 7, 1183-1186 (1962).

35. X. Fu, K. H. Hong, L. J. Chen, and F. X. Kartner, "Performance scaling of high-power picosecond cryogenically cooled rod-type Yb:YAG multipass amplification," J. Opt. Soc. Am. B 30, 2798-2809 (2013).

36. A. Sennaroglu, U. Demirbas, S. Ozharar, and F. Yaman, "Accurate determination of saturation parameters for $\mathrm{Cr}^{4+}$-doped solid-state saturable absorbers," J. Opt. Soc. Am. B 23, 241-249 (2006).

37. T. Y. Fan, "Heat generation in Nd:YAG and Yb:YAG," IEEE J. Quantum Electron. 29, 1457-1459 (1993).

38. D. C. Brown, S. Tornegard, J. Kolis, C. McMillen, C. Moore, L. Sanjeewa, and C. Hancock, "The application of cryogenic laser physics to the development of high average power ultra-short pulse lasers," Appl. Sci. 6, 23 (2016).
39. S. Kim and H. Ledbetter, "Low-temperature elastic coefficients of polycrystalline indium," Mater. Sci. Eng. A 252, 139-143 (1998).

40. R. J. Corruccini and J. J. Gniewek, Thermal Expansion of Technical Solids at Low Temperatures: A Compilation from the Literature (United States Government Printing Office, 1961).

41. I. Tamer, S. Keppler, M. Hornung, J. Korner, J. Hein, and M. C. Kaluza, "Spatio-temporal characterization of pump-induced wavefront aberrations in $\mathrm{Yb}^{3+}$-doped materials," Laser Photon. Rev. 12, 1700211 (2018).

42. V. Pilla, P. R. Impinnisi, and T. Catunda, "Measurement of saturation intensities in ion doped solids by transient nonlinear refraction," Appl. Phys. Lett. 70, 817-819 (1997).

43. V. V. Zelenogorskii and E. A. Khazanov, "Influence of the photoelastic effect on the thermal lens in a YLF crystal," Quantum Electron. 40, 40-44 (2010).

44. Z. L. Zhang, Q. Liu, M. M. Nie, E. C. Ji, and M. L. Gong, "Experimental and theoretical study of the weak and asymmetrical thermal lens effect of Nd:YLF crystal for sigma and pi polarizations," Appl. Phys. B 120, 689-696 (2015).

45. A. Isemann, P. Wessels, and C. Fallnich, "Directly diode-pumped Colquiriite regenerative amplifiers," Opt. Commun. 260, 211-222 (2006).

46. L. M. Frantz and J. S. Nodvik, "Theory of pulse propagation in a laser amplifier," J. Appl. Phys. 34, 2346-2349 (1963).

47. U. Demirbas, "Modelling and optimization of tapered-diode pumped Cr:LiCAF regenerative amplifiers," Opt. Commun. 311, 90-99 (2013).

48. D. V. Seletskiy, S. D. Melgaard, R. I. Epstein, A. Di Lieto, M. Tonelli, and M. Sheik-Bahae, "Local laser cooling of Yb:YLF to $110 \mathrm{~K}$," Opt. Express 19, 18229-18236 (2011)

49. H. W. Bruesselbach, D. S. Sumida, R. A. Reeder, and R. W. Byren, "Low-heat high-power scaling using InGaAs-diode-pumped Yb:YAG lasers," IEEE J. Sel. Top. Quantum Electron. 3, 105-116 (1997).

50. H. Vanherzeele, "Continuous wave dual rod $\mathrm{Nd}$-Ylf laser with dynamic lensing compensation," Appl. Opt. 28, 4042-4044 (1989).

51. E. C. Honea, R. J. Beach, S. C. Mitchell, J. A. Skidmore, M. A. Emanuel, S. B. Sutton, S. A. Payne, P. V. Avizonis, R. S. Monroe, and D. G. Harris, "High-power dual-rod Yb : YAG laser," Opt. Lett. 25 , 805-807 (2000).

52. A. L. Calendron, H. Cankaya, and F. X. Kartner, "High-energy kHz Yb:KYW dual-crystal regenerative amplifier," Opt. Express 22, 24752-24762 (2014)

53. H. Cankaya, A. L. Calendron, C. Zhou, S. H. Chia, O. D. Mucke, G. Cirmi, and F. X. Kartner, " $40-\mu \mathrm{J}$ passively CEP-stable seed source for ytterbium-based high-energy optical waveform synthesizers," Opt. Express 24, 25169-25180 (2016).

54. D. F. Zhang, A. Fallahi, M. Hemmer, X. J. Wu, M. Fakhari, Y. Hua, H. Cankaya, A. L. Calendron, L. E. Zapata, N. H. Matlis, and F. X. Kartner, "Segmented terahertz electron accelerator and manipulator (STEAM)," Nat. Photonics 12, 336-342 (2018).

55. D. F. Zhang, A. Fallahi, M. Hemmer, H. Ye, M. Fakhari, Y. Hua, H. Cankaya, A. L. Calendron, L. E. Zapata, N. H. Matlis, and F. X. Kartner, "Femtosecond phase control in high-field terahertz-driven ultrafast electron sources," Optica 6, 872-877 (2019). 